\title{
Spatially-averaged oscillatory flow over a rough bed
}

\author{
Stephen E. COLEMAN ${ }^{1}$, Vladimir I. NIKORA ${ }^{2}$, \\ and Ted SCHLICKE ${ }^{1}$ \\ ${ }^{1}$ Department of Civil and Environmental Engineering, The University of Auckland, \\ Auckland, New Zealand \\ e-mails: s.coleman@auckland.ac.nz (corresponding author); tedschlicke@yahoo.com \\ ${ }^{2}$ School of Engineering, University of Aberdeen, Aberdeen, United Kingdom \\ e-mail: v.nikora@abdn.ac.uk
}

\begin{abstract}
A rigorous framework involving flow decomposition and averaging is presented, within which the mechanics of rough- (e.g., rippled-) bed oscillatory flows can be better interpreted and understood. Spatiallyaveraged equations for conservation of fluid mass and momentum are developed for analyses of rapidly-changing bed conditions, e.g., for growing ripples. Where repeated observations of the changing bed conditions are available, the ensemble and spatially-averaged versions of these equations can be used for more detailed analyses of the flow dynamics.

The double-averaged (in space and phase or time) equations of mass and momentum conservation are shown to be appropriate for analyses of flows over fixed rough beds and equilibrium ripples. The value of the present framework is highlighted herein by its application to PIVmeasured oscillatory-flow velocities, stresses and vorticities over growing and equilibrium wave-induced intermediate-depth orbital-vortex ripples. In particular, discussions are provided regarding the mechanisms by which gravity-induced and pressure-gradient-induced momentum is transferred to the bed, with the analysis framework naturally and explicitly including the combination of the full range of fluid stresses and boundary form and skin friction drag that is important in defining the flow mechanics.
\end{abstract}

Key words: ripple initiation, ripple growth, equilibrium ripple, double averaging, spatial averaging, PIV, vortex ripple.

(C) 2008 Institute of Geophysics, Polish Academy of Sciences 


\section{INTRODUCTION}

The complex interaction between waves, tides, currents and sediment results in bed forms whose sizes range over several orders of magnitude. The largest of these are tidal sand banks, with amplitudes of tens of metres and wavelengths of several kilometres (Blondeaux 2001). At the other extreme are gravity-wave-induced ripples, several centimetres long. These small-scale bedforms increase friction near the bed (causing wave attenuation at the water surface and flow separation at the bed), and give rise to local increases in both sediment transport and dispersion of passive substances.

The typically-turbulent flow governing fluxes in sand-bed coastal environments, including sediment-transport and mixing processes, varies with time, wave phase and space. The flow may also be strongly influenced by rough-bed heterogeneity, particularly near the bed, e.g. through the presence of bed ripples or gravel particles, which leads to feedback between the bed and the overhead flow. In order to better understand the mechanics of roughbed oscillatory flows, it is helpful to decompose the flow and associated fluxes into constituent components. This approach stems from the classical Reynolds decomposition leading to the establishment of the time- (ensemble-) averaged Reynolds equations from the Navier-Stokes equations, and insightful interpretation of flows based on the averaged equations.

Various authors have undertaken such an approach of flow decomposition and averaging for oscillatory flows. Nielsen (1992) presents a good account of time-averaged, wave-phase-averaged, and turbulent fluctuating components of momentum flux and their interpretation, without considering, however, any effects of spatial variations or averaging. Nielsen (1992) notes that correlations in phase velocities generally dominate fluid stresses, particularly in relation to lesser-magnitude turbulence correlations.

Ranasoma and Sleath (1992) used spatial averaging to analyse measured phase velocities, turbulence intensities, and momentum-flux components. With no velocities measured below ripple crests, spatial averaging was undertaken only above ripple crests. Based on theoretical considerations, Giménez-Curto and Corniero Lera (1996) undertook formal spatial averaging of ensemble-averaged (phase-averaged for purely oscillatory flow) equations of motion. They highlight that spatial variations of flow properties can play a significant role in governing momentum flux, e.g., through boundary drag (skin friction and pressure drag) and form-induced fluid stresses (arising from correlations of spatial variations in ensemble-averaged properties).

In modelling surface-wave motions in the vicinity of a permeable structure, Hsu et al. (2002) utilize Volume-Averaged Reynolds-Averaged NavierStokes (VARANS) equations. They decompose fluid velocity into spatiallyaveraged and varying-in-space ensemble averages and a fluctuating component, but do not include wave-phase terms in velocity decomposition or 
averaged equations of motion (noted by Nielsen (1992) to be significant in regard to fluid stresses). For oscillatory flow through submerged canopies, Lowe et al. (2005a) decompose fluid velocities into a phase- (or time-) andspatially-averaged current, phase-averaged wave components (spatiallyaveraged and varying in space) and a fluctuating component, with their focus being the root-mean-square phase-averaged wave component of velocity. They utilise a depth-averaged form of the phase- and spatially-averaged expression for momentum conservation, with drag incorporating pressure drag (without skin friction effects) and fluid inertia effects for unsteady flow, and with all fluid stresses combined in a single term. Interestingly, they conclude that in-canopy flow is larger for oscillatory flow than for comparable unidirectional flow owing to the different momentum balances established inside the canopies for these flows (Lowe et al. 2005b).

With different authors decomposing flow parameters to varying degrees, utilising varying levels of spatial and temporal averaging, and interpreting oscillatory flows within varying frameworks, this paper provides a general framework involving flow decomposition and averaging within which the mechanics of rough (rippled) bed oscillatory flows can be better interpreted and understood. To this end, the double-averaged (in space and phase or time) equations for oscillatory flow over an equilibrium rough bed are outlined and derived in Section 2. Section 3 presents the equivalent spatialaveraging framework and equations for flow over rapidly-developing sand ripples. Recent investigations of oscillatory flow over intermediate-depth orbital-vortex ripples growing from plane-bed conditions to equilibrium are described in Section 4. Results of these experiments highlighting the validity and value of the framework are presented in Sections 5 and 6, along with interpretation of bed and flow-field structures, dynamics and interaction for the growing and equilibrium orbital-vortex ripples. The paper conclusions are given in Section 7.

\section{DOUBLE-AVERAGED EQUATIONS FOR OSCILLATORY FLOW OVER FIXED ROUGH BEDS AND EQUILIBRIUM RIPPLES}

\subsection{Reynolds decomposition and averaging}

The starting point for analysis of fluid motion is the combination of the continuity and Navier-Stokes equations for instantaneous variables, namely for an incompressible fluid:

$$
\frac{\partial u_{i}}{\partial x_{i}}=0, \quad \frac{\partial u_{i}}{\partial t}+\frac{\partial u_{i} u_{j}}{\partial x_{j}}=g_{i}-\frac{1}{\rho} \frac{\partial p}{\partial x_{i}}+\frac{\partial}{\partial x_{j}}\left(v \frac{\partial u_{i}}{\partial x_{j}}\right),
$$

where $t$ is time, $\rho$ is the fluid density, $g_{i}$ is the component of gravitational acceleration in direction $x_{i}=(x, y, z), u_{i}$ is the $i$ th component of the point vector of instantaneous velocity $(u, v, w), p$ is the point pressure, $v$ is the fluid 
kinematic viscosity, and the tensor notation is used with the Einstein convention, which prescribes a summation over each repeated index.

For turbulent flows with instantaneous velocity expressed as the sum of time-averaged and fluctuating components, namely $u_{i}=\bar{u}_{i}+u_{i}^{\prime}$, the timeaveraged continuity and classical Reynolds equations are derived from (1) by substituting for $u_{i}$ and time averaging, giving:

$$
\frac{\partial \bar{u}_{i}}{\partial x_{i}}=0, \quad \frac{\partial \bar{u}_{i}}{\partial t}+\frac{\partial \bar{u}_{i} \bar{u}_{j}}{\partial x_{j}}=g_{i}-\frac{1}{\rho} \frac{\partial \bar{p}}{\partial x_{i}}-\frac{\partial \overline{u_{i}^{\prime} u_{j}^{\prime}}}{\partial x_{j}}+\frac{\partial}{\partial x_{j}}\left(v \frac{\partial \bar{u}_{i}}{\partial x_{j}}\right) .
$$

\subsection{Averaging oscillatory flows in time or phase}

For flows involving oscillatory components, the instantaneous velocity can further be expressed (e.g., Nielsen 1992) as

$$
\begin{aligned}
& u_{i}(t)=\bar{u}_{i}+\hat{u}_{i}(t)+u_{i}^{\prime}(t), \quad \bar{u}_{i}=\frac{1}{t_{0}} \int_{t_{0}} u_{i}\left(t^{\prime}\right) \mathrm{d} t^{\prime}, \\
& \hat{u}_{i}(t)=\left\{\frac{1}{N_{0}} \sum_{j=0}^{j=N_{0}-1} u_{i}\left[t^{\prime} \equiv t(\bmod T)\right]\right\}-\bar{u}_{i},
\end{aligned}
$$

where $t_{0}$ is the averaging time interval; $\hat{u}_{i}$ is the phase average (relative to $\bar{u}_{i}$ ) over a large number $N_{0}$ of wave periods $T<<t_{0}$; and $t^{\prime} \equiv t(\bmod T)$ relates conditions (over $N_{0}$ waves) at successive times $t^{\prime}$ that are of the same wave phase, i.e. $(t \bmod T)$. An example relation between $u, \bar{u}, \hat{u}$, and $u^{\prime}$ is shown in Fig. 1 for measurement of $u$ at a given position in a periodic flow of period $T$. Pressure $p$ can be similarly decomposed. With the definition of (3), $\overline{\hat{u}_{i}}=\overline{u_{i}^{\prime}}=\widehat{u_{i}^{\prime}}=\overline{\overline{u_{i}} \hat{u}_{j}}=\overline{\overline{u_{i}} u_{j}^{\prime}}=\overline{\hat{u}_{i} u_{j}^{\prime}}=\widehat{\overline{u_{i} u_{j}^{\prime}}}=\widehat{\hat{u}_{i} u_{j}^{\prime}}=0$.

Akin to the Reynolds equations, for flows involving oscillatory motion, the phase-averaged equations for conservation of mass and momentum can be obtained by substituting for $u_{i}$ (and equivalently for pressure $p$ ) from (3) into (1) and phase averaging (with zero mean values) the resulting equation, namely (e.g., Nielsen 1992):

with

$$
\frac{\partial \hat{u}_{i}}{\partial x_{i}}=0, \quad \frac{\partial \hat{u}_{i}}{\partial t}=g_{i}-\frac{1}{\rho} \frac{\partial \hat{p}}{\partial x_{i}}+\frac{\partial \hat{\tau}_{i j} / \rho}{\partial x_{j}},
$$

$$
\hat{\tau}_{i j}=\rho\left(v \frac{\partial \hat{u}_{i}}{\partial x_{j}}-\hat{u}_{i} \hat{u}_{j}-\widehat{u_{i}^{\prime} u_{j}^{\prime}}-\bar{u}_{i} \hat{u}_{j}-\hat{u}_{i} \bar{u}_{j}\right) .
$$

In a similar fashion, the time-averaged equations for conservation of mass and momentum can be obtained by substituting for $u_{i}$ (and equivalently for pressure $p$ ) from (3) into (1) and time averaging the resulting equation, namely (e.g., Nielsen 1992): 


$$
\frac{\partial \bar{u}_{i}}{\partial x_{i}}=0, \quad \frac{\partial \bar{u}_{i}}{\partial t}=g_{i}-\frac{1}{\rho} \frac{\partial \bar{p}}{\partial x_{i}}+\frac{\partial \bar{\tau}_{i j} / \rho}{\partial x_{j}}
$$

with

$$
\bar{\tau}_{i j}=\rho\left(v \frac{\partial \bar{u}_{i}}{\partial x_{j}}-\bar{u}_{i} \bar{u}_{j}-\overline{\hat{u}_{i} \hat{u}_{j}}-\overline{u_{i}^{\prime} u_{j}^{\prime}}\right) .
$$

The time-averaged oscillatory-flow equations of (6) are equivalent to the classical Reynolds equations of (2), with the addition of a fluid stress (i.e., momentum flux component) due to correlations in phase velocities for the wave-induced motion, $\bar{\tau}_{o s c_{i j}}=-\rho \overline{\hat{u}}_{i} \hat{u}_{j}$. Nielsen (1992) notes that of the components of $\bar{\tau}_{i j}, \bar{\tau}_{\text {oscij }}=-\rho \overline{\hat{u}_{i} \hat{u}_{j}}$ is generally dominant, except very close to the bed, notably influencing the shape of $\bar{u}(z)$ profiles.

\subsection{Averaging in time (or phase) and space}

For flows over rough (e.g., rippled) beds, the phase- and time-averaged variables in (4) to (7) can be highly spatially heterogeneous. In contrast, bed roughnesses are typically described using spatially-averaged parameters, and thereby cannot be linked explicitly with local (point) flow properties provided by the time- (or phase-) averaged equations. Spatial (volume) averaging of the time- (or phase-) averaged hydrodynamic variables and equations eliminates this problem, where the averaging volume is defined by the longitudinal, transverse, and vertical length-scales of the roughness elements. In most practical cases of interest, the suitable averaging volume is a thin slab parallel to the bed. The resulting double-averaged (in space and time or phase) equations relate to the time- (or phase-) averaged equations as the latter relate to the Navier-Stokes equations for instantaneous hydrodynamic variables. In the following derivation of double-averaged equations describing oscillatory flow, the approach outlined by Nikora et al. (2007) for rough-bed open-channel and overland flows is extended.

In adopting the double-averaging approach for description of oscillatory flows over rough beds, each component in $u_{i}=\bar{u}_{i}+\hat{u}_{i}+u_{i}^{\prime}$ (Fig. 1) will vary spatially over a rough bed and can be further decomposed into spatiallyaveraged and spatially-fluctuating components, i.e., $\bar{u}_{i}=\left\langle\bar{u}_{i}\right\rangle+\tilde{u}_{i}$, $\hat{u}_{i}=\left\langle\hat{u}_{i}\right\rangle+\tilde{\hat{u}}_{i}$, and $u_{i}^{\prime}=\left\langle u_{i}^{\prime}\right\rangle+\widetilde{u_{i}^{\prime}}$. For the simplified case of no subdivision of turbulent fluctuations $u_{i}^{\prime}$, the instantaneous velocity can then be generally expressed as

$$
u_{i}=\left\langle\bar{u}_{i}\right\rangle+\tilde{u}_{i}+\left\langle\hat{u}_{i}\right\rangle+\tilde{\hat{u}}_{i}+u_{i}^{\prime}, \quad\left\langle\bar{u}_{i}\right\rangle=\frac{1}{V_{f}} \iiint_{V_{f}} \bar{u}_{i} \mathrm{~d} V, \quad\left\langle\hat{u}_{i}\right\rangle=\frac{1}{V_{f}} \iiint_{V_{f}} \hat{u}_{i} \mathrm{~d} V,
$$

where the angle brackets denote the spatial averaging of flow variables within the spatial domain occupied by fluid, the wavy overbar denotes the spatial 


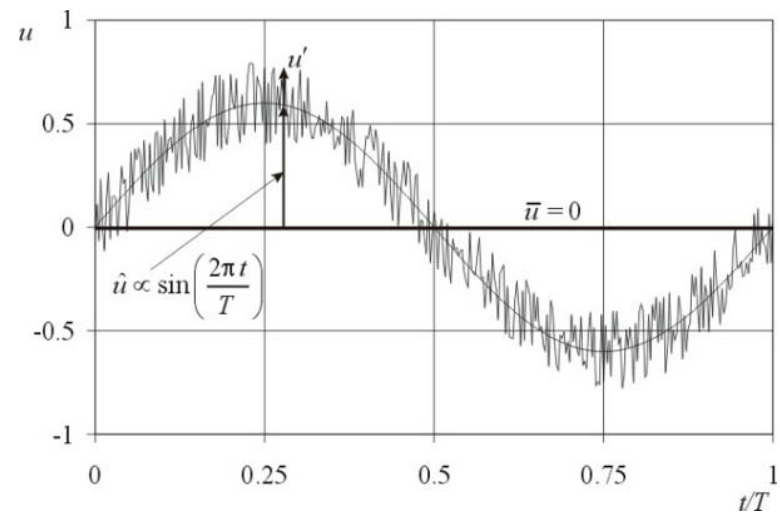

Fig. 1 Principal velocity decomposition at a point in purely oscillatory flow of $\bar{u}=0$. For plane-bed oscillatory flow, $u$ can be decomposed as $u=\bar{u}+\hat{u}+u^{\prime}$ (Section 2.2), where $u=\bar{u}+u^{\prime}$ for non-oscillatory flow (Section 2.1). For spatiallyvarying flow over a fixed or equilibrium rough bed, $u$ can be further decomposed as $u=\bar{u}+\hat{u}+u^{\prime}=\langle\bar{u}\rangle+\tilde{u}+\langle\hat{u}\rangle+\tilde{\hat{u}}+u^{\prime}$ (Section 2.3). For spatially-varying flow over a rapidly-changing rough bed, $u$ can be decomposed as $u=\langle u\rangle+\breve{u}$ (Section 3.1), or $u=\langle\hat{u}\rangle+\tilde{\hat{u}}+u^{\prime} \quad$ where multiple observations of the bed and flow development are available to enable ensemble averaging and determination of the turbulent fluctuating component $u^{\prime}$ (Section 3.2).

fluctuations of $\bar{u}_{i}$ and $\hat{u}_{i}$, and $V_{f}=$ volume occupied by fluid within a fixed region $R$ centred at level $z$ with the total averaging volume $V_{0}$. In this paper, we also assume $\left\langle\left\langle\bar{u}_{i}\right\rangle\right\rangle=\left\langle\bar{u}_{i}\right\rangle,\left\langle\tilde{u}_{i}\right\rangle=0,\left\langle\left\langle\hat{u}_{i}\right\rangle\right\rangle=\left\langle\hat{u}_{i}\right\rangle$, and $\left\langle\tilde{\hat{u}}_{i}\right\rangle=0$.

The operation of spatial averaging for the flow region below roughness crests is not trivial because it does not always commute with time and spatial differentiation. In order for the time- (or phase-) averaged equations to be averaged in the space domain, averaging theorems (based on the transport theorem and the spatial-averaging theorem) need to be applied. The appropriate averaging theorems (extended from Nikora et al. 2007) for fixed rough beds and equilibrium ripples (that can fluctuate as the wave passes) are

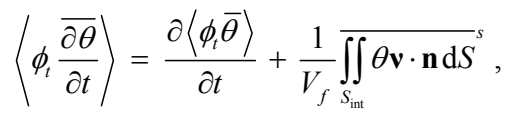

$$
\begin{aligned}
& \left\langle\phi_{p} \frac{\widehat{\partial \theta}}{\partial t}\right\rangle=\frac{\partial\left\langle\phi_{p} \hat{\theta}\right\rangle}{\partial t}+\frac{1}{V_{f}} \iint_{S_{\mathrm{int}}} \theta \mathbf{v} \cdot \mathbf{n} \mathrm{d} S,
\end{aligned}
$$

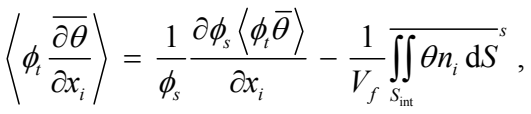




$$
\left\langle\phi_{p} \frac{\widehat{\partial \theta}}{\partial x_{i}}\right\rangle=\frac{1}{\phi_{s}} \frac{\partial \phi_{s}\left\langle\phi_{p} \hat{\theta}\right\rangle}{\partial x_{i}}-\frac{1}{V_{f}} \iint_{S_{\text {int }}} \theta n_{i} \mathrm{~d} S,
$$

where $\theta=$ flow variable (e.g., scalar or vector component) defined in the fluid but not at points occupied by the roughness elements (where it can also be assumed to be zero); $\phi_{t}=t_{f} / t_{0}$ and $\phi_{p}=N_{f} / N_{0}$ are time and phase analogues of the roughness geometry (or porosity) function $\phi_{s}=V_{f} / V_{0} ; t_{0}$ and $N_{0}$ are total averaging time interval and total number of time moments involved in phase averaging, respectively, including periods when the spatial points are intermittently occupied by fluid and roughness elements (e.g., by moving bed particles); $t_{f}$ is averaging time interval equal to the sum of the time periods when a spatial point under consideration is occupied by fluid only; $N_{f}$ is the number of time moments involved in phase averaging when a spatial point under consideration is occupied by fluid only; $\phi_{s}$ is assumed to be invariant (or slowly-varying) in time for equilibrium ripples; $\boldsymbol{v}=$ bedsurface velocity; $n_{i}$ is the $i$ th component of the unit vector normal to the surface element $\mathrm{d} S$ and directed into the fluid; $S_{\text {int }}=$ extent of water-bed interface within the averaging volume; and the superscript $s$ refers to the superficial spatial integral (over volume $V_{0}$ rather than $V_{f}$ ). Above the roughness crests, the averaging region $R$ is completely occupied by fluid $\left(\phi_{s}=\phi_{t}=\phi_{p}=1\right)$, while below them it is intersected by roughness elements and it may become multiply connected.

Averaging the equations of (1) in space and time or phase (with zero mean values), with $u_{i}$ decomposed in the form of (8) as developed from (3), and implementing no slip and kinematic boundary conditions, we then obtain the following double-averaged equations:

$$
\begin{gathered}
\frac{\partial \phi_{s}\left\langle\hat{u}_{i}\right\rangle}{\partial x_{i}}=0, \\
\frac{\partial\left\langle\hat{u}_{i}\right\rangle}{\partial t}=g_{i}-\frac{1}{\rho \phi_{s}} \frac{\partial \phi_{s}\langle\hat{p}\rangle}{\partial x_{i}}+\frac{1}{\phi_{s}} \frac{\partial}{\partial x_{j}}\left(\phi_{s} \frac{\left\langle\hat{\tau}_{i j}\right\rangle}{\rho}\right)+\frac{1}{\rho} \frac{1}{V_{f}}{\widehat{\iint_{S_{\text {int }}} p n_{i} \mathrm{~d} S}}^{s}-\frac{1}{V_{f}} \iint \bar{S}_{S_{\text {int }}}\left(v \frac{\partial u_{i}}{\partial x_{j}}\right) n_{j} \mathrm{~d} S \\
\frac{\partial \phi_{s}\left\langle\bar{u}_{i}\right\rangle}{\partial x_{i}}=0, \\
\frac{\partial\left\langle\bar{u}_{i}\right\rangle}{\partial t}=g_{i}-\frac{1}{\rho \phi_{s}} \frac{\partial \phi_{s}\langle\bar{p}\rangle}{\partial x_{i}}+\frac{1}{\phi_{s}} \frac{\partial}{\partial x_{j}}\left(\phi_{s} \frac{\left\langle\bar{\tau}_{i j}\right\rangle}{\rho}\right)+\frac{1}{\rho} \frac{1}{V_{f}}{\overline{\iint_{\text {int }} p n_{i} \mathrm{~d} S}}^{s}-\frac{1}{V_{f}} \iint_{S_{\text {int }}}\left(v \frac{\partial u_{i}}{\partial x_{j}}\right) n_{j} \mathrm{~d} S
\end{gathered}
$$

where any fluctuations in equilibrium ripples have been neglected as minor $\left(\phi_{t}=\phi_{p}=1\right)$, the theorems of (10) and (12) have been used to derive (13) and (14), and (9) and (11) have been used to derive (15) and (16).

The final two terms of (14) and (16) represent spatially- and (phase- or) time-averaged form drag and surface (skin friction) drag per unit fluid vo- 
lume (these terms being zero above roughness crests). These equations can then be seen to describe the fluid-flow transfer of gravity- and pressuregradient-induced momentum to the boundary, where it is removed through form drag and skin friction. The momentum transfer components of $\left\langle\hat{\tau}_{i j}\right\rangle$ and $\left\langle\bar{\tau}_{i j}\right\rangle$ include:

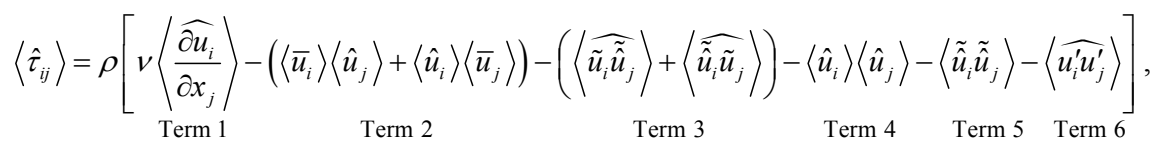

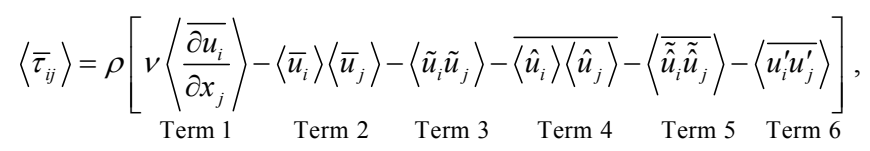

where in each case, Term 1 represents viscous stresses, Term 6 is turbulent stresses, Term 4 stands for wave-phase stresses (arising from correlations in phase velocities), Terms 3 and 5 represent form-induced stresses (arising from correlations in spatial fluctuations of phase-averaged and timeaveraged velocities, e.g., Giménez-Curto and Corniero Lera 1996 and Nikora et al. 2007), and Term 2 describes convective transfer of momentum. The formulation of (13) to (18) explicitly includes the full combination of fluid stresses of different origins (including wave-phase stresses and form-induced stresses) and boundary form and skin friction drag highlighted to varying degrees in previous studies (e.g., in the respective works of Nielsen 1992 and Giménez-Curto and Corniero Lera 1996).

The value of the framework of (8) and (13) to (18) lies in being able to effectively dissect flows over plane beds and equilibrium ripples to consider the principal mechanisms acting to govern the flow mechanics. This is highlighted in terms of experimentally-measured flows in Section 6.

\section{OSCILLATORY FLOW OVER RAPIDLY-DEVELOPING RIPPLES}

\subsection{Spatial averaging}

The previous section presents flow decomposition and double-averaged equations of motion for fixed or equilibrium beds of conditions that are violated for ripples developing rapidly from flat-bed conditions to equilibrium magnitudes. For developing ripples, the starting point for analysis of fluid motion is again the combination of the continuity and Navier-Stokes equations for instantaneous variables given by (1) for an incompressible fluid. Without flow measurements for repeated ripple developments (refer to Section 3.2), effectively no time or phase averaging is possible for this rapidly-changing 
bed that is of a development time comparable to the wave period. Instantaneous velocities can then only be spatially decomposed, i.e.,

$$
u_{i}=\left\langle u_{i}\right\rangle+\breve{u}_{i}, \quad\left\langle u_{i}\right\rangle=\frac{1}{V_{f}} \iiint_{V_{f}} u_{i} \mathrm{~d} V,
$$

where the inverted-arch overbar denotes deviation of an instantaneous variable at a given spatial point from its instantaneous spatially-averaged value. Without time or phase averaging, instantaneous velocity then has two components, $\left\langle u_{i}\right\rangle$ and $\breve{u}_{i}$. Applying to (1) the averaging theorems for instantaneous variables, namely (e.g., Whitaker 1999):

$$
\left\langle\frac{\partial \theta}{\partial t}\right\rangle=\frac{1}{\phi_{s}} \frac{\partial \phi_{s}\langle\theta\rangle}{\partial t}+\frac{1}{V_{f}} \iint_{S_{\mathrm{itt}}} \theta \mathbf{v} \cdot \mathbf{n} \mathrm{d} S
$$

and

$$
\left\langle\frac{\partial \theta}{\partial x_{i}}\right\rangle=\frac{1}{\phi_{s}} \frac{\partial \phi_{s}\langle\theta\rangle}{\partial x_{i}}-\frac{1}{V_{f}} \iint_{S_{\text {int }}} \theta n_{i} \mathrm{~d} S
$$

expanding $u_{i}$ as per (19), and implementing no slip and kinematic boundary conditions, the spatially-averaged conservation equations are derived as

$$
\begin{gathered}
\frac{\partial \phi_{s}\left\langle u_{i}\right\rangle}{\partial x_{i}}=0, \\
\frac{1}{\phi_{s}} \frac{\partial \phi_{s}\left\langle u_{i}\right\rangle}{\partial t}=g_{i}-\frac{1}{\rho \phi_{s}} \frac{\partial \phi_{s}\langle p\rangle}{\partial x_{i}}+\frac{1}{\phi_{s}} \frac{\partial}{\partial x_{j}}\left(\phi_{s} \frac{\left\langle\tau_{i j}\right\rangle}{\rho}\right)+\frac{1}{\rho V_{f}} \iint_{S_{\text {int }}} p n_{i} \mathrm{~d} S-\frac{1}{V_{f}} \iiint_{S_{\text {int }}}\left(v \frac{\partial u_{i}}{\partial x_{j}}\right) n_{j} \mathrm{~d} S,
\end{gathered}
$$

The latter equation again describes the fluid-flow transfer of gravity- and pressure-gradient-induced momentum to the boundary, where it is removed through form drag and skin friction. The momentum flux components in this case comprise:

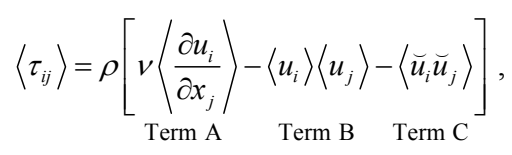

where Term A represents viscous stresses, Term C is form-induced stresses (arising from correlations in spatial fluctuations of instantaneous velocities), and Term B combines convective transfer of momentum with turbulence correlations and correlations in phase velocities. Unlike the equilibrium-bed analysis of Section 2.3, the constituent components of Terms B and C (all of which change with time as the boundary develops) cannot be separated owing to rapid ripple development preventing any decomposition and averaging in terms of time or wave phase. 


\subsection{Ensemble and spatial averaging}

To provide information on phase-averaged and turbulent quantities for a rapidly-developing bed, the development process can be repeatedly observed and the flow quantities ensemble- and spatially-averaged for each point in time. The ensemble averaging is thereby equivalent to phase averaging (at a particular time $t$ ) as the bed develops. With this approach, velocities for the developing ripples can be decomposed as

$$
u_{i}=\left\langle\hat{u}_{i}\right\rangle+\tilde{\hat{u}}_{i}+u_{i}^{\prime}, \quad\left\langle\hat{u}_{i}\right\rangle=\frac{1}{V_{f}} \iiint_{V_{f}} \hat{u}_{i} \mathrm{~d} V,
$$

where $u_{i}=\hat{u}_{i}+u_{i}^{\prime}$ is the sum of ensemble-averaged $\hat{u}_{i}$ (averaged at the wave phase at time $t$ ) and turbulent fluctuating $u_{i}^{\prime}$ parts, and angled brackets and the wavy overbar denote further decomposition into spatially-averaged and spatially-fluctuating terms.

The ensemble- and spatial-averaging theorems for flow over developing ripples are

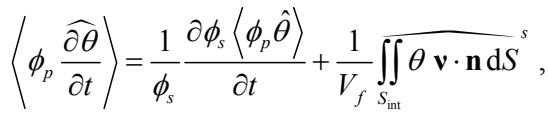

$$
\begin{aligned}
& \left\langle\phi_{p} \frac{\widehat{\partial \theta}}{\partial x_{i}}\right\rangle=\frac{1}{\phi_{s}} \frac{\partial \phi_{s}\left\langle\phi_{p} \hat{\theta}\right\rangle}{\partial x_{i}}-\frac{1}{V_{f}} \iint_{S_{\mathrm{mt}}} \theta n_{i} \mathrm{~d} S .
\end{aligned}
$$

Applying (26) and (27), expanding $u_{i}$ as per (25), and implementing no slip and kinematic boundary conditions, the ensemble- (equivalent to phase at a given time) and spatially-averaged equation for momentum conservation is then derived from (1) as

$$
\frac{1}{\phi_{s}} \frac{\partial \phi_{s}\left\langle\hat{u}_{i}\right\rangle}{\partial t}=g_{i}-\frac{1}{\rho \phi_{s}} \frac{\partial \phi_{s}\langle\hat{p}\rangle}{\partial x_{i}}+\frac{1}{\phi_{s}} \frac{\partial}{\partial x_{j}}\left(\phi_{s} \frac{\left\langle\hat{t}_{i j}\right\rangle}{\rho}\right)+\frac{1}{\rho V_{f}} \iint_{S_{\text {int }} p n_{i} \mathrm{~d} S}^{s}-\frac{1}{V_{f}} \iint_{S_{\text {int }}}\left(v \frac{\partial u_{i}}{\partial x_{j}}\right) n_{j} \mathrm{~d} S,
$$

where any fluctuations in repeated measurements have been neglected as minor $\left(\phi_{p}=1\right)$, the last two terms represent spatially- and phase-averaged form (pressure) drag and surface (skin friction) drag per unit fluid volume, and the momentum flux components of $\left\langle\hat{\tau}_{i j}\right\rangle$ are given by

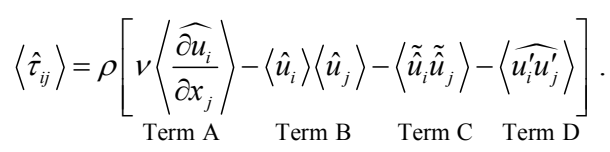

In (29), Term A represents viscous stresses, Term C is form-induced stresses (arising from correlations in spatial fluctuations of ensemble-averaged veloc- 
ities), Term B combines convective transfer of momentum and wave-phase (arising from correlations in phase velocities) stresses, and Term D is turbulent stresses. Adopting this framework of flow decomposition and averaging, the development of key components of flow velocities, stresses and vorticity can be investigated for growing ripples, as illustrated in the analyses of Section 5.

\section{EXPERIMENTAL FACILITIES, PROCEDURES AND PARAMETERS 4.1 Experimental design}

In a series of twenty-one experiments, Coleman et al. (2005) simultaneously measured the profile of a mobile-bed orbital-vortex ripple train together with the associated overhead flow (in space and time) as the ripples developed from plane-bed conditions to equilibrium magnitudes. Gravity-wave-induced fluid velocities were measured using the non-intrusive technique of particle image velocimetry (PIV), while the ripple profile was ascertained using edge-detection methods for the recorded images. By adopting this measurement approach, the way in which the flow and bed profile change with time can be related, thereby enhancing understanding of nonlinear interactions between them.

Of the twenty-one experiments of Coleman et al. (2005), Test f1.00h150 is analysed herein to illustrate application of the framework of Sections 2 and 3 to equilibrium and developing beds. The analysed test was of mean water depth $h=0.15 \mathrm{~m}$, and was of waves of length $\lambda=1.09 \mathrm{~m}$, amplitude $a=0.031 \mathrm{~m}$, and frequency $\omega=2 \pi \mathrm{rad} / \mathrm{s}$. These waves are of $h / \lambda=0.14$, confirming intermediate depths $(0.05<h / \lambda<0.5)$ of orbital near-bed fluid motion for the test. The linear-wave theoretical near-bed orbital diameter for this run, $2 A_{b}=2 a /[\sinh (k h)]$, is $0.064 \mathrm{~m}$. For the test equilibrium ripples, of height $\eta_{e}=0.0085 \mathrm{~m}$ and length $\lambda_{e}=0.042 \mathrm{~m}$ (steepness $\eta_{e} / \lambda_{e}=0.2$ ),

$$
\lambda_{e}=1.3 A_{b} .
$$

This ratio (for a mobility number of $\psi=\left(A_{b} \omega\right)^{2} /[(s-1) g d]=12.5$, where $g$ is the gravitational acceleration) is in close agreement with values obtained by other researchers for orbital-vortex ripples (e.g., Raudkivi 1998, Admiraal et al. 2006), indicating that the ripples formed in these experiments are orbital rather than anorbital in nature (discussed further in Coleman et al. 2005, which also provides values of additional flow parameters).

The Coleman et al. (2005) experiments were conducted in a wave flume of length $L=5 \mathrm{~m}$ and width $0.15 \mathrm{~m}$ (Fig. 2). At one end of the flume, there was a sinusoidally-oscillating piston (of no active absorption processes) to generate gravity waves (travelling right to left, i.e., in the negative $x$ direc- 
(a)
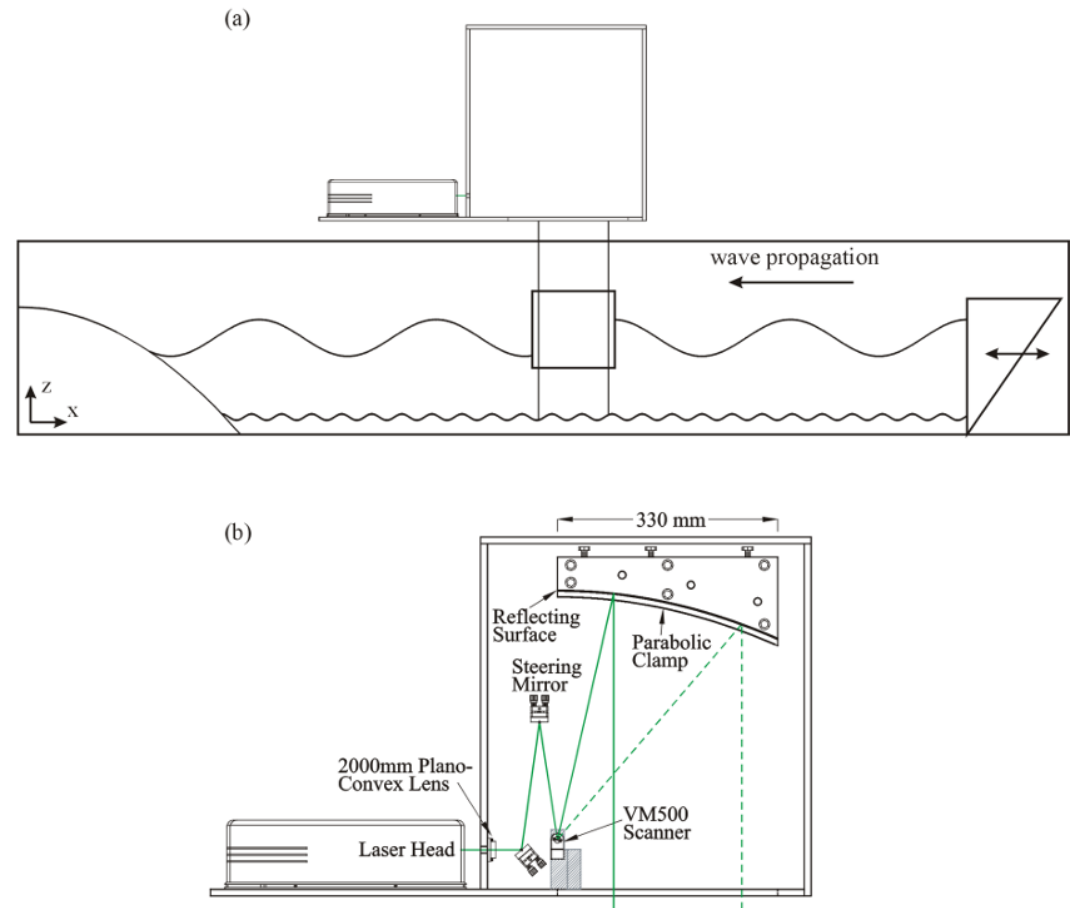

Fig. 2: (a) Wave flume and particle image velocimetry (PIV) installation, and (b) details of PIV system.

tion). A porous shingle beach of parabolic profile was constructed at the other end of the flume to dissipate wave energy and minimise wave reflections (tests using Method III of Isaacson 1991 giving an estimated reflection coefficient of $6 \%$ ).

The angular wave frequency $\omega$ for an experiment was equal to that of the wavemaker oscillation, while the wave amplitude $a$ is non-linearly related to the wavemaker stroke amplitude (Hughes 1993). The amplitude and frequency of the generated waves were varied across the experiments of Coleman et al. (2005), with their values chosen such that: (a) the wavelength $\lambda$ was significantly less than the length of the tank $(L k>>2 \pi$, where angular wave number $k=2 \pi / \lambda$ ); (b) the waves were in intermediate or shallow water ( $k h \approx 1<\pi$, Svendsen and Jonsson 1976, for the present tests); (c) the waves were non-breaking ( $a k<0.32$, Longuet-Higgins 1997); and (d) the waves would move the sediment. The mobile bed was $0.015 \mathrm{~m}$ deep when flattened and consisted of uniform (geometric standard deviation of sizes $\sigma_{g} \leq 1.3$ ) quartz sediment of diameter $d=0.2 \mathrm{~mm}$ and submerged relative density $(s-1)=1.65$, where $s=\rho_{s} / \rho, \rho_{s}$ is the sediment density, and $\rho$ is the water density.

As the wavemaker was warming up for each test, a gate positioned in the flume in front of the wavemaker was initially closed to avoid sediment 
movement by waves of increasing frequency. When the wavemaker was running at its steady state, the gate barrier was rapidly raised at the start of PIV image recording, and waves began propagating down the flume. It was found from preliminary experiments that ripples could form at either end of the tank, and migrate towards the middle. In order to investigate repeatable ripple evolution, and avoid simply measuring the flow above essentially fully-developed ripples traversing into the lightsheet, the initially-plane sediment bed was scored with a small indentation across the flume width prior to the commencement of the experiment to trigger ripple formation within the lightsheet. From the velocity measurements, it was noted that transient effects were significant for the first five wave periods after the gate was raised. After this time and prior to ripple generation (approximately 20 wave periods thereafter), the velocities at a particular point were observed to be essentially invariant from one wave period to the next. Near the bed, the flow changed with time as ripples formed and grew.

\subsection{PIV system}

For PIV measurement of velocities, the water was seeded with Dantec S-HGS silver-coated hollow glass spheres of $10 \mu \mathrm{m}$ diameter. The tank was illuminated from above by a "Scanning Beam Box" ("SBB", designed at The University of Auckland), which consists of (Schlicke et al. 2007): a frequency-doubled $5 \mathrm{~W}$ continuous-wave $(\mathrm{CW}) \mathrm{Nd}: \mathrm{YVO}_{4}$ laser; a convex lens to control the beam diameter and ensure the beam waist is at the region of interest; and a computer-controlled GSI Lumonics VM500 galvanometer mirror located at the focal point of a plane parabolic mirror. The galvanometer mirror (Fig. 2) sweeps the beam along the parabola, from which it is reflected vertically downwards, resulting in a rapidly-scanning laser lightsheet approximately $0.1 \mathrm{~m}$ wide and $1 \mathrm{~mm}$ thick. A streamlined glass wave-guide was used to pass the lightsheet through the moving water surface without undesirable refractions (Schlicke et al. 2005). The lightsheet was positioned $0.03 \mathrm{~m}$ from the flume wall to aid image clarity (suspended sediments giving decreasing clarity of PIV tracers with increasing distance from the wall), while avoiding viscous-drag wall effects.

For the present ripple tests, the scanning beam was synchronised to a Basler A504k high-speed camera (8 bit monochrome camera, of up to $1280 \times 1024$ pixels and with a CMOS sensor), such that each exposure of the camera began as the beam started sweeping in the downstream direction. The beam scan time was equal to the exposure time of the camera, and was set to $3 \mathrm{~ms}$. The 'fly-back' time of the galvanometer, the time taken for the beam to return to its starting position, was $2 \mathrm{~ms}$. Fifty pairs of images were thereby recorded each second; the time between images in a pair being $5 \mathrm{~ms}$, with a separation between image pairs of $20 \mathrm{~ms}$. The image data was transferred directly to the harddrive of a RAID configured PC, enabling recording 
at the desired $100 \mathrm{fps}$ for over fifteen minutes duration for the tests. For each test, images were recorded at this peak rate for the first two minutes, by which time the ripples were approximately at equilibrium, then for $2 \mathrm{~s}$ every $10 \mathrm{~s}$ for the next 4 minutes. The imaged flow area measured approximately $0.085 \mathrm{~m}$ by $0.085 \mathrm{~m}$.

The system of flow visualization used for these tests was designed (Schlicke et al. 2007) to satisfy a range of generic desires for hydraulics applications, including: flow-field resolution at frequencies of up to $200 \mathrm{~Hz}$ (with continuous recording durations of over 8 minutes); linear beam scan velocity (minimising variations in the intensity distribution of the sheet); the rotating mirror always being positioned at the parabolic-mirror focus; lightsheet width and beam scan velocity being easily and independently adjustable; and the system facilitating frame-straddling techniques for lightsheet imaging.

\subsection{PIV-based flow analyses}

The field of flow velocities from an image pair was obtained from image cross-correlation (FFT based), with an interrogation window size of $32 \times 32$ pixels, and a grid spacing of 16 pixels. The pixel data were normalised (to a zero mean, with unit standard deviation) to reduce bias error. The correlation peak was located to sub-pixel accuracy using a Gaussian peak-fitting algorithm. The resulting velocity vector spacing was $1.4 \mathrm{~mm}$ in the horizontal and vertical directions for the interrogation window used. Spurious vectors (outliers) were identified using a local-median comparison scheme based on the velocity magnitude. Outlier velocity values were assigned the local median velocity components. No additional smoothing of velocities was performed. In terms of uncertainties in velocity estimates, lower limit particledisplacement uncertainties were estimated as of the order of 0.05 pixels. A more typical displacement uncertainty of several times this magnitude (e.g., 0.25 pixels) combined with a particle displacement away from the bed of around 12 pixels under a wave crest gives an uncertainty in measured velocities of approximately $2 \%$. This uncertainty will be larger when the particle displacement is less, i.e., towards the bed, although these uncertainties are random and are thereby reduced by the averaging procedures adopted herein. The images were edge detected at the bottom (Coleman et al. 2005) to determine bed-profile development in terms of ripple amplitude and wavelength.

The gravity waves for each test propagated in the $-x$ direction (Fig. 2), with $z$ denoting the height above the mean bed level (MBL). Wave phase $\phi=0^{\circ}$ is defined for a wave crest being directly overhead. The data analyses follow the framework described in Sections 2 and 3 for interpretation of rough (rippled) bed oscillatory-flow mechanics. 
For the present tests of two-dimensional flows and flow measurements, the horizontal and vertical velocities are denoted by $u$ and $w$, respectively, and the spatially- and (phase- or) time-averaged expressions for the fluid stresses of Section 2.3 reduce to:

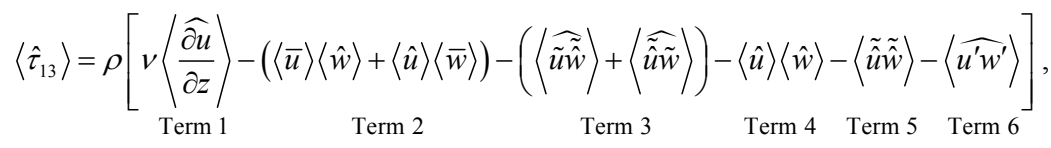

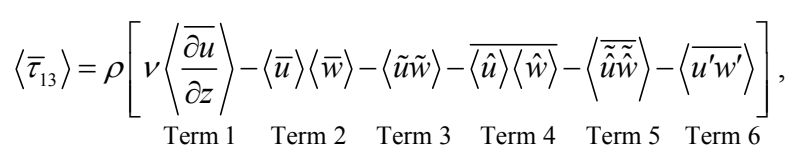

for plane and equilibrium beds of $u_{i}=\left\langle\bar{u}_{i}\right\rangle+\tilde{u}_{i}+\left\langle\hat{u}_{i}\right\rangle+\tilde{\hat{u}}_{i}+u_{i}^{\prime}$. For the developing ripples, the Section 3.2 fluid stresses become

$$
\left\langle\hat{\tau}_{13}\right\rangle=\rho\left[v\left\langle\frac{\widehat{\partial u}}{\partial z}\right\rangle-\langle\hat{u}\rangle\langle\hat{w}\rangle-\langle\tilde{\hat{u}} \tilde{\hat{w}}\rangle-\left\langle\widehat{u^{\prime} w^{\prime}}\right\rangle\right],
$$

where each test analysed was repeated a number of times and velocities were decomposed as $u_{i}=\left\langle\hat{u}_{i}\right\rangle+\tilde{\hat{u}}_{i}+u_{i}^{\prime}$, with $\hat{u}_{i}$ being an ensemble average of spatially-averaged and spatially-fluctuating parts. In-plane phase-averaged flow vorticity $\omega_{y}$ (positive anticlockwise) was calculated for the developing and equilibrium beds using

$$
\hat{\omega}_{y}=\frac{\partial \hat{w}}{\partial x}-\frac{\partial \hat{u}}{\partial z} .
$$

It is important to note that the PIV-image cross-correlations leading to the present velocity estimates did not discriminate between the neutrallybuoyant PIV seeding particles and suspended sediments, with the implicit assumption that measured velocities represent fluid velocities. The flow decompositions of (8) and (25) indicate that the respective equilibrium- (or plane-) bed and developing-bed vertical-velocity estimates of $\langle\bar{w}\rangle$ and $\langle\hat{w}\rangle$ are consequently biased low by up to the sediment fall velocity of $w_{s}=0.025 \mathrm{~m} / \mathrm{s}$. This bias, which is dependent on local sediment concentration, is discussed further in terms of velocities and the stress components of (31) to (33) in Sections 5 and 6 for the developing and equilibrium beds.

For two reasons, there is a phase difference across the vector map obtained directly from an image pair (e.g., the upper-flow vectors in Fig. 3a). The lesser (due to the high beam scan velocity of $28 \mathrm{~m} / \mathrm{s}$ ) source of difference arises because the laser beam is scanning as the wave is propagating, resulting in the different vector columns corresponding to the flow at different phases. Secondly, there is a phase difference due to the finite size of the measurement area relative to the wavelength of the gravity wave. The combined phase lag $\Delta \phi$ is given by 


$$
\Delta \phi=k \Delta x+\omega \Delta t=k \Delta t\left(v_{s}+v_{p}\right),
$$

where $\Delta x$ is the width of the vector map (approximately $0.085 \mathrm{~m}$ ), $\Delta t$ is the time taken for the beam to scan across the vector map $(\approx 3 \mathrm{~ms}), v_{s}=\Delta x / \Delta t$ is the scan velocity of the laser beam $(\approx 28 \mathrm{~m} / \mathrm{s})$ and $v_{p}=\omega / k$ is the phase velocity of the wave $(\approx 1 \mathrm{~m} / \mathrm{s})$. For the focus experiment analysed herein (Test f1.00h150 of Coleman et al. 2005), the wave length $\lambda=2 \pi / k$ was $1.09 \mathrm{~m}$, the wave height was $0.062 \mathrm{~m}$, and the wave frequency $\omega=2 \pi \mathrm{rad} / \mathrm{s}$. Hence, the phase lag $\Delta \phi$ across a vector map determined directly from an image pair for this test is roughly $30^{\circ}$ (e.g., the upper-flow vectors in Fig. 3a).

In order to facilitate the flow decompositions leading to (31)-(34), vector maps corresponding to specific wave phases (e.g., Fig. 3b) were generated from the maps incorporating phase lags that were obtained from the raw images (e.g., Fig. 3a). To do this, the vertical velocity components of consecutive vector maps were examined to determine wave phase for each vector column independently. The time at which this component changes from positive (upwards) to negative (downwards) corresponds to a wave crest passing overhead, $\phi=0^{\circ}$, and was located to subframe accuracy using interpolation. Other phases were determined by interpolating between successive occurrences of $\phi=0^{\circ}$. The effect of this interpolation process is demonstrated in Fig. 3, which corresponds to $\phi=270^{\circ}$. Figure 3a shows the vector map obtained by cross-correlating appropriate raw image pairs; it can be seen that the phase at the right-hand side leads that at the left, because the wave is propagating from right to left. Figure $3 \mathrm{~b}$ was obtained by locating the phase of each column independently, and combining columns of like phase. For Fig. 3b, it can be seen that the upper-flow vectors away from the bed are pointing in approximately the same direction, and thus correspond to the same phase. Although Fig. 3b is a combination of same-phase vectors for the purposes of flow decomposition and is not a physically-occurring flow field for the tested waves, analysis herein of bed and flow dynamics is not adversely distorted by the discussion of such figures (e.g., Figs. 5 and 10), with resulting interpretations being confirmed by the other presented figures.

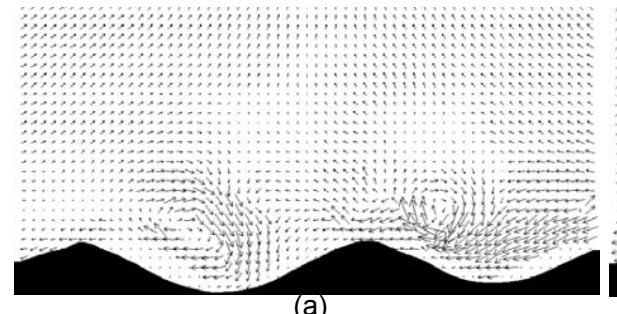

(a)

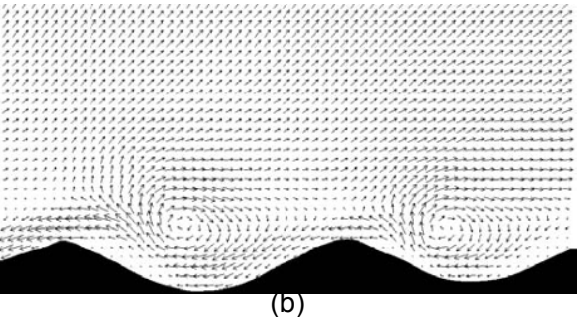

(b)

Fig. 3. Velocity fields obtained from (a) basic correlation, and (b) phase correction (ripple length $=0.042 \mathrm{~m}$ ). 
The decompositions of velocities and the expressions of (31)-(34) were evaluated based on the compiled "same-phase" vector maps of Fig. 3b. For the plane-bed and equilibrium-bed configurations, phase averaging was carried out over ten consecutive wave cycles. For the developing ripples, ensemble averaging was carried out over five run repeats (aligned with a common wavemaker phase at gate removal). These numbers of wave cycles used for the averaging are unfortunately low. Analysis results are consequently illustrative to a degree, although confidence in the results increases as turbulent contributions to the oscillatory flow reduce relative to contributions from orbital motions (e.g. refer to Section 6.3). Improved analyses of larger averaging numbers are anticipated for future measurements using the present framework. Spatial averaging was carried out over fluid-occupied space along velocity vector rows (i.e., in thin slabs parallel to the mean bed level, Nikora et al. 2007, above and below ripple crests), and over a single crest-to-crest wavelength. The slowly-migrating crest positions for these analyses were determined by analysis of the edge-detected bed profiles for the recorded images. The validity of the averaging procedures adopted herein is confirmed by the agreement, highlighted in the results sections, of theoretical expectations with plane-bed measurements of oscillatory flows.

Spatial-averaging is an effective means of data-reduction for rough-bed flows (e.g. Giménez-Curto and Corniero Lera 1996, Nikora et al. 2001, 2007), particularly for the large volumes of data generated by PIV measurements. More importantly, this approach allows the significance of flow features due to wave-phase, bedform and turbulent-fluctuation effects to be compared. Sections 5 and 6 present such analyses for developing and equilibrium ripples, respectively. It is useful to note that if the spatial-averaging domain is extended laterally to include all scales of fluid motion, then the spatial averaging becomes averaging in space and time (e.g., over all phases for domains encompassing orbital diameters), and double-averaged results are obtained (e.g., Coleman and Nikora 2008).

\section{DEVELOPING RIPPLES}

\subsection{Bed development}

For the analysed test, ripple wavelength, $\lambda_{r}$, and crest-to-trough height, $\eta_{r}$, are shown as a function of time in Fig. 4a for the single ripple that developed from the initial indentation. The time origin corresponds to five wave cycles after the opening of the in-flume gate, i.e., after the initial transient effects were determined to have died away. Ripple steepness, defined here as $\eta_{r} / \lambda_{r}$, is shown as a function of time in Fig. $4 \mathrm{~b}$.

At first, the waves acted to flatten and broaden the initial twodimensional bed indentation such that the steepness decreased. At this stage, 
(a)

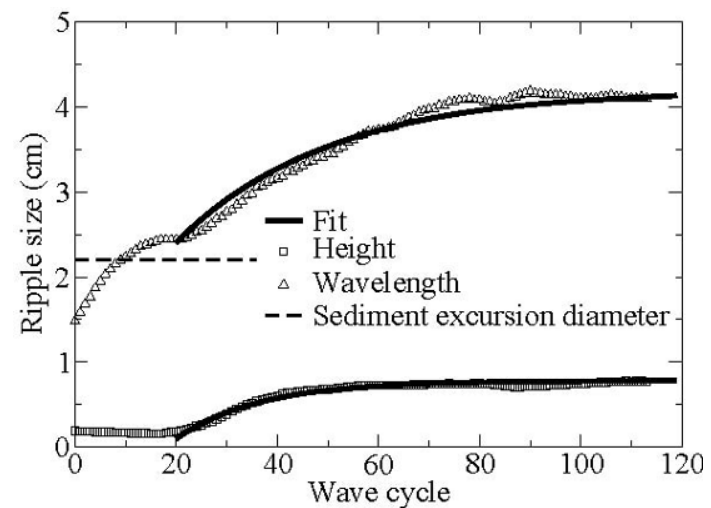

(b)

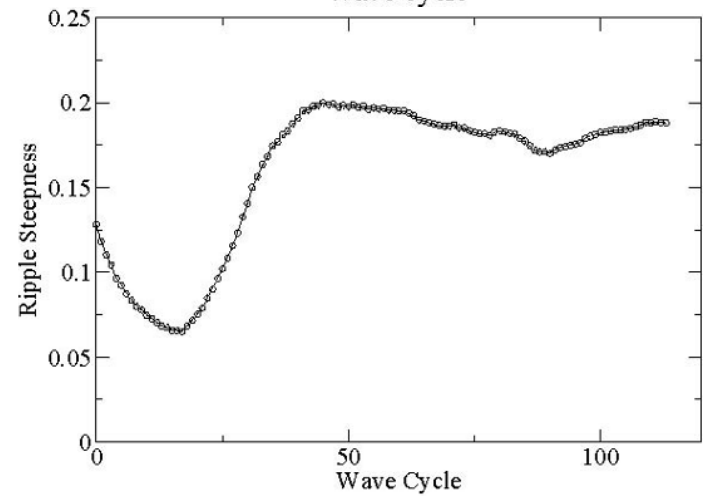

Fig. 4. Ripple (a) height and wavelength ("Fit" is the ripple-growth relation of Coleman et al. 2005), and (b) steepness as functions of wave cycle.

the bedform was simply a depression, entirely beneath the mean bed level. After approximately 20 wave cycles, the bedform changed nature: on either side of the initial indentation, crests began to appear and grow in amplitude, and the bedform was then considered to be a single ripple. The amplitude and wavelength of this ripple then both began to increase, with amplitude growing more rapidly than wavelength, such that the ripple steepness increased steadily. During this period of growth, troughs, and then additional ripples, formed both up and downstream of the crests either side of the initial indentation. The period of rapid growth of the central ripple continued for around 20 wave cycles, after which time the amplitude levelled out. The wavelength continued to increase, resulting in a slight decrease in the steepness. After around 90 wave cycles, the primary ripple was at equilibrium, with ripple wavelength and height at approximately constant values of $\lambda_{e}=$ $0.042 \mathrm{~m}$ and $\eta_{e}=0.0085 \mathrm{~m}$, respectively. The ripples were two-dimensional in nature throughout their development.

Lofquist (1978) suggests that the number of wave cycles $N$ required to reach equilibrium is of the order of $A / d$; approximately 270 for the present experiment. While the central ripple reached equilibrium magnitudes of wave- 
length and amplitude after only $N=90$, the ripple train upstream and downstream continued to develop. The experiment was continued up to $N=360$ with no further increase in $\lambda_{r}$ or $\eta_{r}$. During the additional time, the number of ripples in the train increased until the entire bed of the flume was rippled, with each ripple of height $\eta_{r}$ and wavelength $\lambda_{r}$. In ripple development experiments, therefore, the time to equilibrium magnitudes, and average ripple characteristics at any time, can depend on the length of the ripple train being considered.

Ripple heights grow from essentially zero magnitudes. Finite initial ripple heights of $\eta_{i}=0.001 \mathrm{~m}$ at $t=0$ in Fig. $4 \mathrm{a}$ are due to ripples being generated from an initial artificial bed deformity (indentation) for the run. The initial ripple wavelength $\lambda_{i}$ (at $N=20$ ) of Fig. 4 a is of the order of 0.6 times the final equilibrium wavelength $\lambda_{e}=0.042 \mathrm{~m}$, consistent with the indications of Voropayev et al. (1999), Faraci and Foti (2002), Davis et al. (2004), and Coleman et al. (2005). Figure 4a highlights the good agreement of the measured data with the ripple-growth relation of Coleman et al. (2005), where coefficient values of $C=0.03$ and 0.06 were adopted for application of the growth relation to the present ripple lengths and heights. Also shown is the linkage between initial ripple wavelength and sediment excursion diameter (based on the orbital fluid motion) highlighted in Coleman et al. (2005).

\subsection{Plane-bed flow}

The plane-bed flow measurements prior to ripple generation (wave cycle $N<20$, Fig. 4a) can be decomposed as $u_{i}=\left\langle\bar{u}_{i}\right\rangle+\tilde{u}_{i}+\left\langle\hat{u}_{i}\right\rangle+\tilde{\hat{u}}_{i}+u_{i}^{\prime}$ (Section 2.3), with the flow thereby described by (13) to (18), (31) and (32), and with near-bed vertical-velocity estimates $\langle\bar{w}\rangle$ biased low by suspended sediments.

When the bed deformation consists solely of the initial perturbation on an otherwise plane bed, the fluid motion is approximately elliptical and can be reasonably described by plane-bed wave theory. Graphs comparing ensemble- and spatially-averaged horizontal and vertical velocities and total shear stress as functions of phase are shown in Fig. 5, where a wave phase of $0^{\circ}$ corresponds to a wave crest overhead. Also shown are the predictions of second-order Stokes' theory for flow over a plane bed, for which (e.g., Dean and Dalrymple 1991),

$$
\begin{aligned}
& u(\phi)=-a \omega\left[\frac{\cosh k(h+z)}{\sinh k h}\right] \cos \phi-\frac{3}{4}\left[\frac{a^{2} \omega k \cosh 2 k(h+z)}{\sinh ^{4} k h}\right] \cos 2 \phi=\hat{u}(\phi) \approx\langle\hat{u}\rangle(\phi), \\
& w(\phi)=-a \omega\left[\frac{\sinh k(h+z)}{\sinh k h}\right] \sin \phi-\frac{3}{4}\left[\frac{a^{2} \omega k \sinh 2 k(h+z)}{\sinh ^{4} k h}\right] \sin 2 \phi=\hat{w}(\phi) \approx\langle\hat{w}\rangle(\phi),
\end{aligned}
$$




$$
\tau(\phi)=-\rho\langle\hat{u}\rangle(\phi)\langle\hat{w}\rangle(\phi)=\hat{\tau}(\phi) \approx\langle\hat{\tau}\rangle(\phi)
$$

and vorticity is everywhere zero. In (36) to (38), $z$ is measured from the mean free-surface, and the waves of phase $\phi=k x+\omega t$ travel in the negative $x$ direction over a plane bed. Assuming turbulent effects to be relatively minor, the instantaneous results of (36) to (38) are equivalent to phaseaveraged values. Further assuming the spatial-averaging volume to be much smaller than the wave length, the predictions of (36) to (38) approximate phase- and spatially-averaged values, as indicated above.

In our analysed test, the Ursell parameter value of $2 a \lambda^{2} / h^{3}=22$ is towards the upper-limit value of 26 recommended for applicability of the conventionally-adopted equations of (36) to (38) (e.g., Dean and Dalrymple 1991). Recognising this, and that standard wave theories may struggle to accurately describe the present scale of waves, the Fig. 5 agreement of the present plane-bed measurements (averaged between wave numbers $N=0$ and $N=20$ ) with the wave theory of (36) to (38) can be considered to be rea-

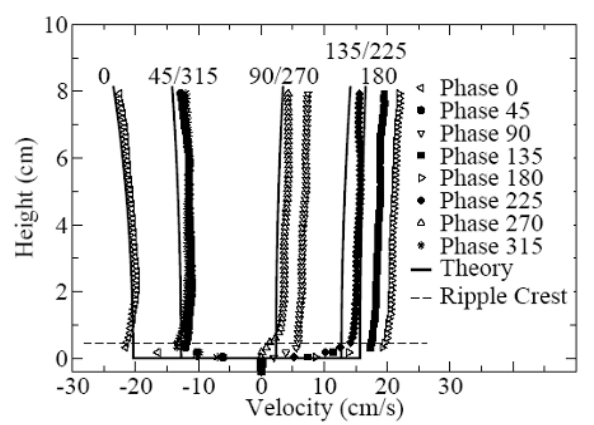

(a)

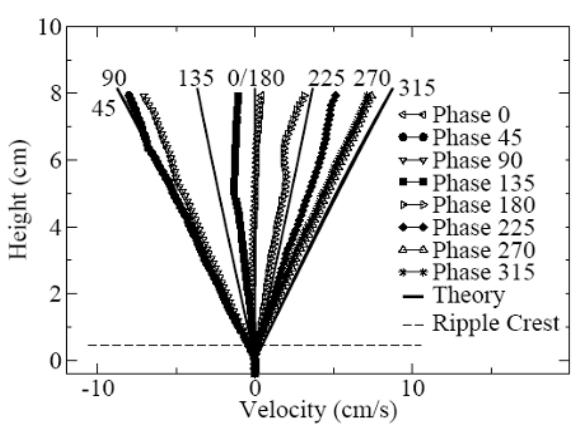

(b)

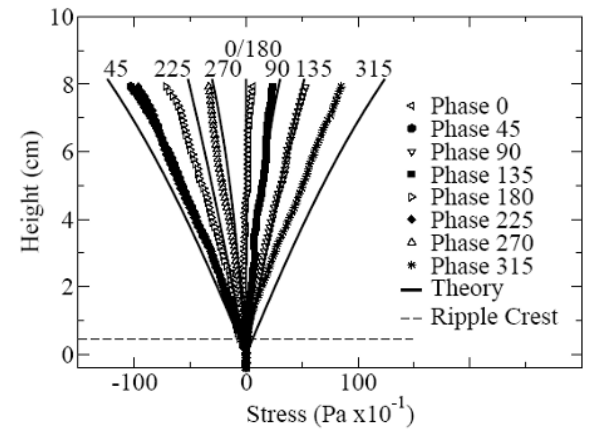

(c)

Fig. 5. Plane-bed conditions. Phase- and spatially-averaged (a) horizontal velocity $\langle\hat{u}\rangle$, (b) vertical velocity $\langle\hat{w}\rangle$, and (c) total shear stress $\langle\hat{\tau}\rangle$ as functions of height (relative to mean bed level) and phase. "Theory" is from (36)-(38) for plane-bed conditions. "Ripple Crest" shown is at equilibrium. 
sonable. The comparable trends and magnitudes provide confidence in the respective performances of the velocity-measurement system and analysis procedures adopted for the testing (including valid tracking of the flow by the PIV seeding particles). They also support the views that (a) the generated waves were fully developed by the time they reached the measuring section; (b) transient effects due to gate removal initiating wave propagation along the flume were dissipated prior to ripple initiation and growth; (c) the wave guide used to pass the PIV lightsheet through the moving water surface did not adversely affect wave-induced flow fields (and thereby generated ripples); and (d) wave reflection effects were not significant for the tests. Planebed theory is further compared in Sections 5 and 6 with flows over rough beds to verify far-field rough-bed measurements and to illustrate rippled-bed effects on flow dynamics.

\subsection{Flow development with ripple growth}

These repeatedly-observed flow measurements for developing ripples can be decomposed as $u_{i}=\left\langle\hat{u}_{i}\right\rangle+\tilde{\hat{u}}_{i}+u_{i}^{\prime}$ (Section 3.2), with the flow thereby described by (28), (29) and (33), and with near-bed vertical-velocity estimates $\langle\hat{w}\rangle$ biased low by suspended sediments.

\section{Flow Fields}

Sample vector maps, depicting the flow at $\phi=0^{\circ}$ and $270^{\circ}$ are shown in Fig. 6, where the velocity vectors are superimposed on flow vorticity, shaded from white $\left(-0.1 \times 10^{3} \mathrm{~s}^{-1}\right)$ to dark grey $\left(0.1 \times 10^{3} \mathrm{~s}^{-1}\right)$. Four different times are shown, defined in terms of number of wave cycles $N(N=0$ being defined when the initial transient effects had died away): (1) $N=20$, the stage at which the initial indentation begins to transform to a ripple; (2) $N=30$, when ripple growth rate reaches a maximum (Fig. 4); (3) $N=40$, at which time the ripple development slows down; and (4) $N=100$, by which stage the central ripple has reached its equilibrium amplitude and wavelength.

Figure 6 highlights that for the initial bed disturbance, when the oscillatory flow changes direction, around $\phi=270^{\circ}$ (and $90^{\circ}$ ), small vortices are formed on the lee slope of the artificial bed perturbation. These small vortices are confined to a thin region near the bed, but as the ripple grows in steepness, these vortices begin to shed upwards into the main body of fluid. This vortex formation and shedding occurs every half-cycle; in these experiments it was observed that minor recirculation (drift) patterns set up in the flume resulted in the vortex structures being more pronounced in the second half of the wave cycle, results of this half cycle (of $\phi=180^{\circ}-270^{\circ}-360^{\circ}$ ) being presented herein. Ripple growth is facilitated by the separation-cell vortices moving sediment from the trough to the crests of the ripples. Equi- 

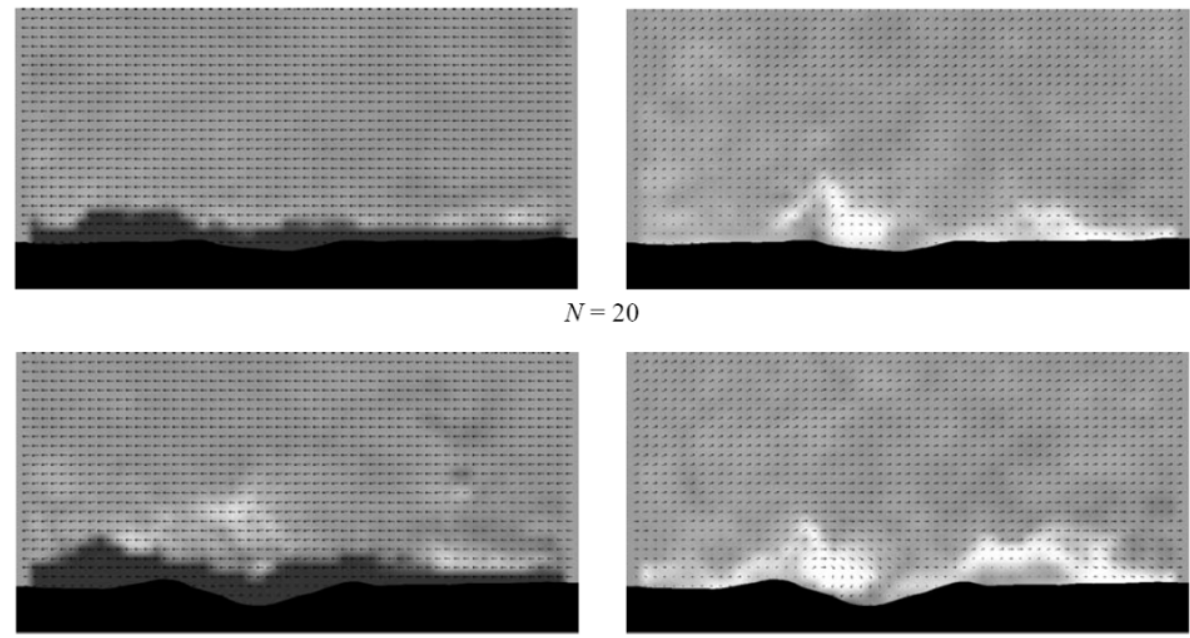

$N=30$
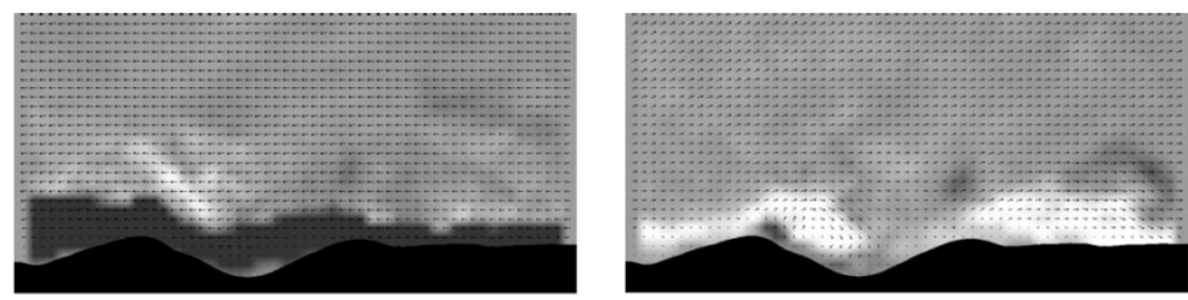

$N=40$
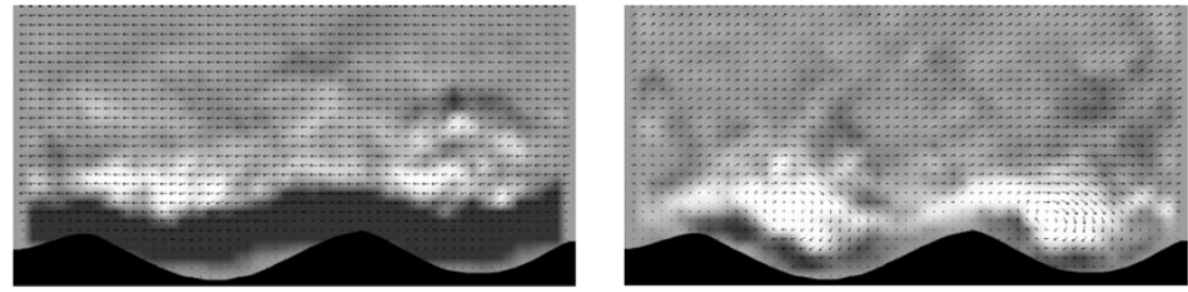
$\phi=0^{\circ}$
$\phi=270^{\circ}$
$50 \overrightarrow{\mathrm{mm}} / \mathrm{s}$
$20 \overrightarrow{\mathrm{mm}} / \mathrm{s}$

0.1

$-0.02$

0

0.02

0.04

Fig. 6. Developing ripples. Velocity and vorticity fields for $\phi=0^{\circ}$ and $270^{\circ}$ at various stages of ripple development. Respective vector and $\hat{\omega}_{y}\left(\mathrm{~s}^{-1} \times 10^{3}\right)$ scales are highlighted. Waves propagate right to left, with the position-based results of Figs. 7 and 8 being midway between the ripple trough and the immediately-downstream (to the left above) crest.

librium magnitudes are achieved when this process is balanced by gravity pulling the sediment down the ripple slopes. 
Phase $\phi=0^{\circ}$ can be seen in Fig. 6 to correspond to a skimming flow, with a strong shear layer following the bed profile. As indicated above, the reversing flow of $\phi=270^{\circ}$ involves vortex motion between ripple crests, this motion strengthening as the ripples grow, with a thin shear layer developing immediately adjacent to the bed (Fig. 6). Subsequent assessment herein of flow over developing ripples is principally in terms of that occurring at $\phi=0^{\circ}$ and $270^{\circ}$.

\section{Velocities}

The spatially-averaged and spatially-fluctuating components of the phaseaveraged horizontal and vertical velocities are shown in Figs. 7 and 8 for $\phi=270^{\circ}$ and $0^{\circ}$, respectively. The fluctuating components in these graphs correspond to a position midway between the ripple trough and the crest
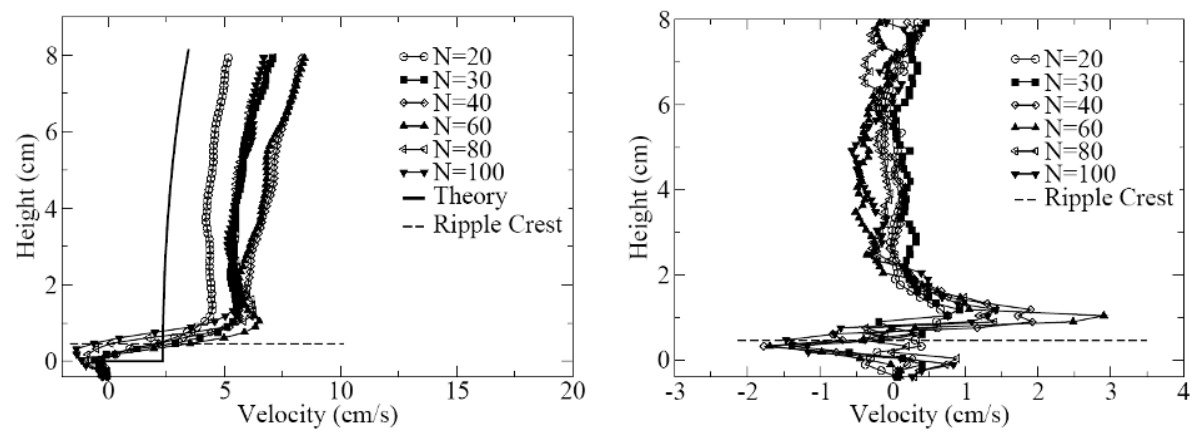

$\langle\hat{u}\rangle$
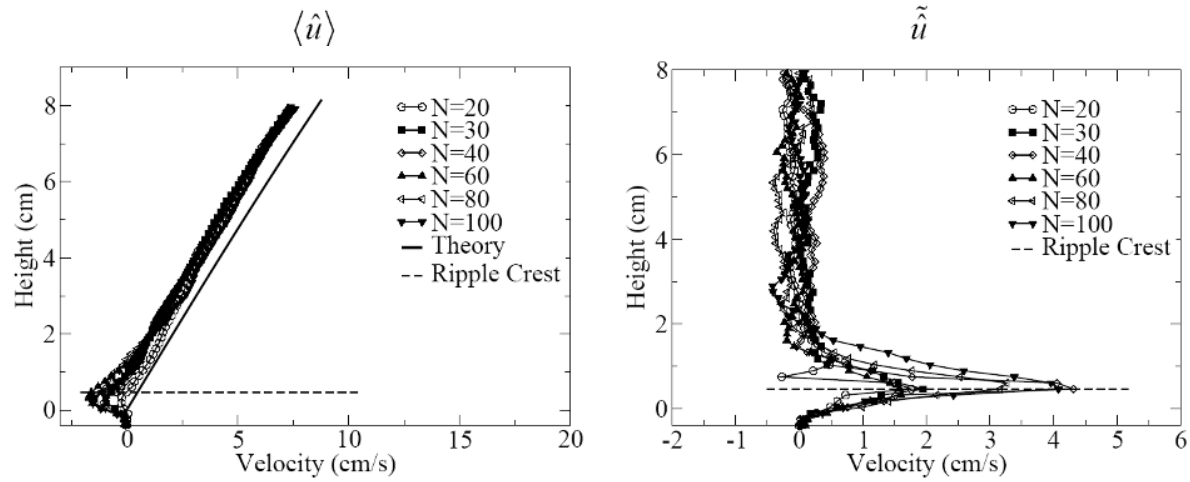

$\langle\hat{w}\rangle$

$\tilde{\hat{w}}$

Fig. 7. Developing ripples. Spatially-averaged and spatially-fluctuating ensembleaveraged horizontal and vertical velocity components as functions of height (relative to mean bed level) and time for $\phi=270^{\circ}$. "Theory" is from (36) and (37) for planebed conditions. "Ripple Crest" shown is at equilibrium. The spatially-fluctuating terms correspond to a position midway between the ripple trough and the immediately-downstream crest (Fig. 6). 
immediately downstream (i.e., at the mean bed level of the negative bed slope in Fig. 6). The results of both Figs. 7 and 8 reflect velocity perturbations becoming more significant and extending further into the flow as ripples grow.
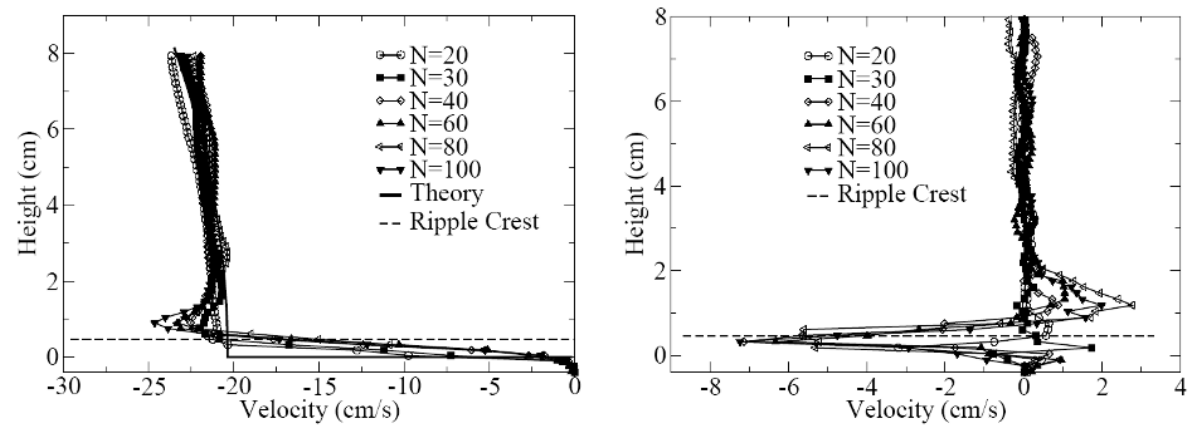

$\langle\hat{u}\rangle$

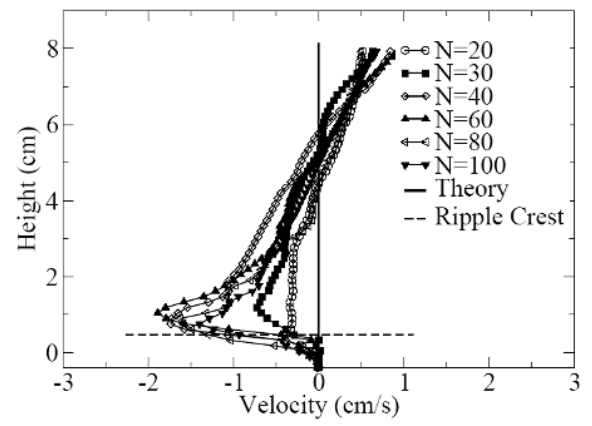

$\tilde{\hat{u}}$

$\langle\hat{w}\rangle$

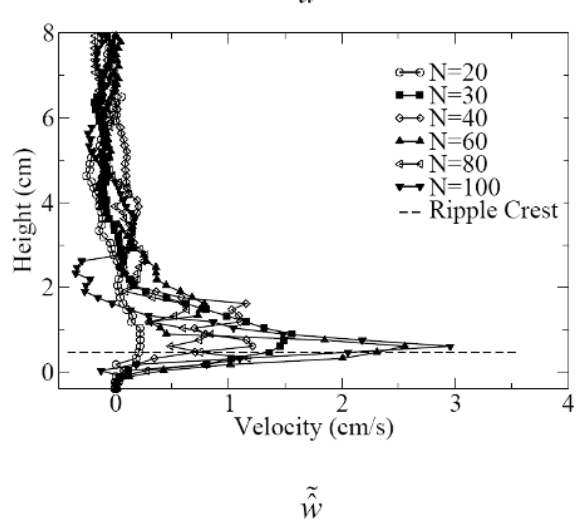

Fig. 8. Developing ripples. Spatially-averaged and spatially-fluctuating ensembleaveraged horizontal and vertical velocity components as functions of height (relative to mean bed level) and time for $\phi=0^{\circ}$. "Theory" is from (36) and (37) for plane-bed conditions. "Ripple Crest" shown is at equilibrium. The spatially-fluctuating terms correspond to a position midway between the ripple trough and the immediatelydownstream crest (Fig. 6).

Neglecting bed friction, the theoretical plane-bed horizontal velocity $u=\langle\hat{u}\rangle$ decays slowly with depth. From Figs. 7 and 8 , it can be seen that above the immediate vicinity of the flat bed, $\langle\hat{u}\rangle$ is reasonably approximated by plane-bed theory, particularly for $\phi=0^{\circ}$. At $\phi=270^{\circ}$ (Fig. 7), $\langle\hat{u}\rangle$ increases with height in the upstream direction (positive $x$ ) near the start of the experiment. As the rippled bed develops, a negative, downstream peak appears immediately above the bed, changing sign around the ripple crest level, i.e., at the ripple amplitude $a_{r}$, and becoming a positive peak. The velocity then falls off again before increasing slowly with height. The local bed-in- 
duced flow acceleration towards and over the ripple crest is also apparent for $\phi=0^{\circ}$.

For both $\phi=270^{\circ}$ and $\phi=0^{\circ}$ (Figs. 7 and 8), $\langle\hat{w}\rangle$ over the flat bed at $N=20$ is in agreement with plane-bed theory away from the bed, increasing approximately linearly with height for $\phi=270^{\circ}$, and approximately equal to zero for $\phi=0^{\circ}$. For the developing bed at both phases, $\langle\hat{w}\rangle$ has a negative peak centred around the level of the ripple crest, indicating a net downward motion over the ripple wavelength. As indicated in Section 4.3, these negative vertical velocities reflect the effect of the sediment settling velocity, with this peak and the associated downward flux generally increasing with time as suspended-sediment motions increase.

For $\phi=270^{\circ}$, the clockwise vortex forming over the lee slope and centred at the level of the ripple crest (Fig. 6) is the dominant flow feature over the ripple wavelength. The spatial fluctuations $\tilde{\hat{u}}$ of Fig. 7 clearly show the velocity peaks at the top and bottom of this vortex, with $\tilde{\hat{w}}$ consisting of near-bed positive perturbations, peaking at the crest level, that reflect the upward flow of the vortex structure at this location (the mean bed level on the negative bed slope of Fig. 6). Above three equilibrium ripple amplitudes $a_{e}$ from the mean bed level $\left(z=3 a_{e}=3 \eta_{e} / 2=0.0128 \mathrm{~m}\right)$, the rippled bed has little effect on the flow and $\tilde{\hat{u}}$ and $\tilde{\hat{w}}$ are approximately zero.

For $\phi=0^{\circ}, \tilde{\hat{u}}$ has a peak of downstream velocity immediately above the bed (Fig. 8), consistent with flow acceleration up the bed slope at this phase (Fig. 6). The fluctuating component $\hat{w}$ has a positive upward peak, again reflecting flow acceleration up the slope at this phase (Fig. 6).

\section{Vorticity}

Ensemble-, given by (34), and spatially-averaged vorticities for $\phi=270^{\circ}$ and $0^{\circ}$ are shown in Figs. 9a and 9b, respectively, where vorticity is positive anticlockwise. For plane-bed conditions $(N \leq 20)$, vorticity approaches the plane-bed wave-theory expectation of zero everywhere, except near the bed where high vorticity occurs within the thin boundary shear layer. For developing ripples at $\phi=270^{\circ}$, there is a small peak of positive vorticity immediately adjacent to the bed due to the local vertical gradient of the horizontal velocity, $\partial \hat{u} / \partial z$. Between the mean bed level, $z=0$, and $z=2 a_{r}$, there is a large negative (clockwise) peak due to the vortex present there (Fig. 6). Between $2 a_{r}$ and $3 a_{r}$, there is another small positive peak of vorticity, which corresponds to the residual anti-clockwise vortex formed a half-wave cycle previously having been shed upwards into the flow. Above this, the vorticity is approximately zero, as predicted by plane-bed wave theory. The strength of the principal vortex between ripples can be seen to grow as the ripples develop (Figs. 6 and 9). 


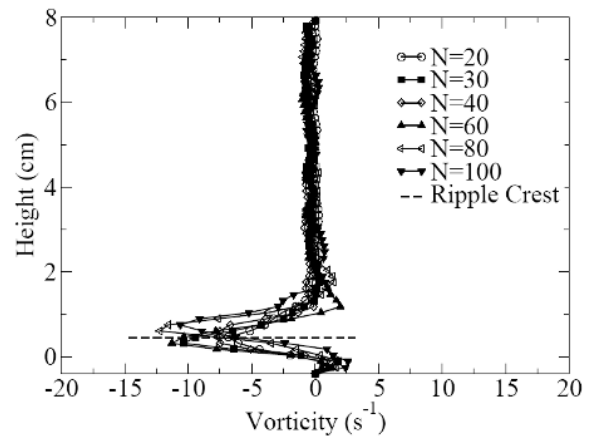

(a)

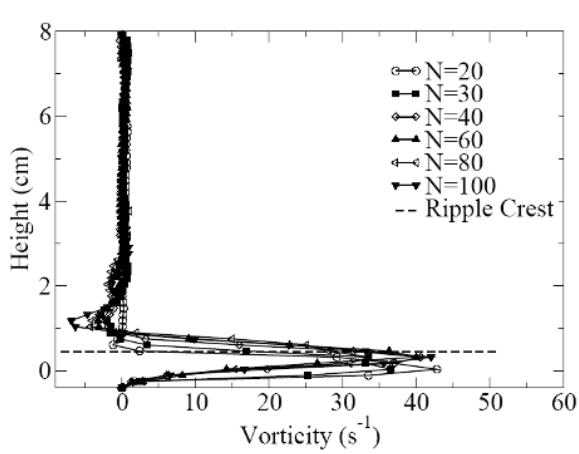

(b)

Fig. 9. Developing ripples. Ensemble- and spatially-averaged vorticity $\left\langle\hat{\omega}_{y}\right\rangle$ as a function of height (relative to mean bed level) and time for: (a) $\phi=270^{\circ}$ and (b) $\phi=0^{\circ}$. "Ripple Crest" shown is at equilibrium.

The vorticity distribution at $\phi=0^{\circ}$ is dominated by the large velocity gradient in the strong near-bed shear layer (Fig. 6). This layer is thinnest when the bed is essentially flat, increasing in thickness as the bed deforms (Fig. 6). Just above the ripple crest, the vorticity has a smaller, negative (clockwise) peak, which corresponds to the vortex structure prominent at $\phi=270^{\circ}$ being shed upwards and downstream (Fig. 6). Shear-layer-induced vorticity can be seen to exceed that arising due to the ripple-generated vortices. Near-bed peak vorticity remains of opposite phase to $\hat{u}$ (Figs. 6 to 9), reflecting the dominance of the shear-layer generated vorticity. Again, outerflow vorticity tends to zero, as predicted by plane-bed wave theory.

\section{Stresses}

The wave-phase component $-\rho\langle\hat{u}\rangle\langle\hat{w}\rangle$ of ensemble- and spatially-averaged shear stress given by (33) is shown in Fig. 10 as a function of height and time at $\phi=315^{\circ}$ and $0^{\circ}$ (of expected peak and minimum stresses, Fig. 5).

This shear-stress component was selected as it is shown below that this component dominates the total stress over the flow depth for equilibrium ripples. The shear stresses of Fig. 10 can be seen to reflect the appropriate combinations of phase-averaged velocities (e.g., Fig. 8 for $\phi=0^{\circ}$ ). For both phases, the wave-phase stresses approximate plane-bed theoretical predictions, increasing linearly with height for $\phi=315^{\circ}$, and approximately equal to zero for $\phi=0^{\circ}$. For the developing bed at both phases, the stresses have an accentuated negative peak centred around the level of the ripple crest. As indicated in Section 4.3, these accentuated negative values reflect the artificial effect of the sediment settling velocity. 


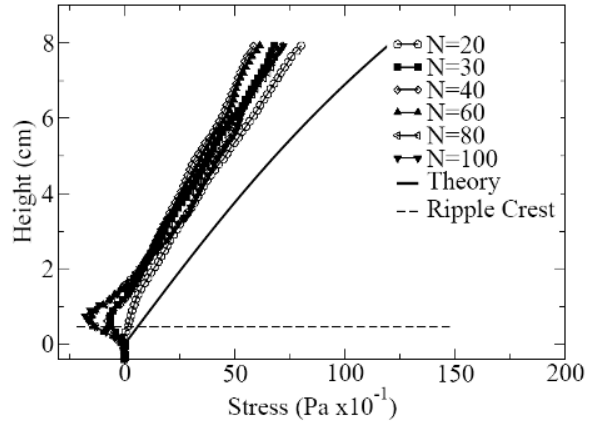

(a)

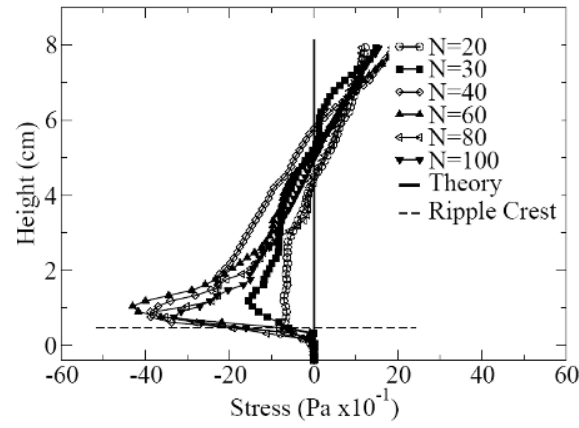

(b)

Fig. 10. Developing ripples. Ensemble- and spatially-averaged shear stress term $-\rho\langle\hat{u}\rangle\langle\hat{w}\rangle$ as a function of height (relative to mean bed level) and time for: (a) $\phi=315^{\circ}$ and (b) $\phi=0^{\circ}$. "Theory" is from (38) for plane-bed conditions. "Ripple Crest" shown is at equilibrium.

\section{EQUILIBRIUM-RIPPLE FLOW STRUCTURE AND DYNAMICS}

These flow measurements can be decomposed as $u_{i}=\left\langle\bar{u}_{i}\right\rangle+\tilde{u}_{i}+\left\langle\hat{u}_{i}\right\rangle+\tilde{\hat{u}}_{i}+u_{i}^{\prime}$ (Section 2.3), with the flow thereby described by (13) to (18), (31) and (32), and with near-bed vertical-velocity estimates $\langle\bar{w}\rangle$ biased low by suspended sediments.

\subsection{Flow fields}

A series of vector maps, corresponding to the flow at phases separated by $45^{\circ}$, is shown in Fig. 11 to illustrate flow features above the bed of equilibrium ripples for the present test. The maps are shaded to illustrate vorticity $\omega_{y}$, with the lightest greyscale corresponding to $-0.1 \times 10^{3} \mathrm{~s}^{-1}$ (clockwise) and the darkest to $+0.1 \times 10^{3} \mathrm{~s}^{-1}$ (anti-clockwise).

Phase $\phi=0^{\circ}$ corresponds to a maximum $u$ component in the $-x$ direction, and a minimum in $w$. At this phase, there is a strong band of vorticity, due to the large gradient in the horizontal velocity in the skimming boundary-layer flow near the bed. As $\phi$ increases, a near-bed recirculation occurs in the ripple trough, which develops into an anti-clockwise vortex (of positive vorticity). At $\phi=90^{\circ}, u$ changes direction and this vortex is swept over the ripple crest, dissipating as a new shear layer forms of opposite sign. The process then essentially repeats in the other direction, though minor recirculation patterns set up in the flume (e.g., Fig. 12c) result in the vortex structures in Fig. 11 being more defined at $\phi=270^{\circ}$ than at $\phi=90^{\circ}$ (asymmetric vortex structure being further discussed in van der Werf et al. 2007). Above 


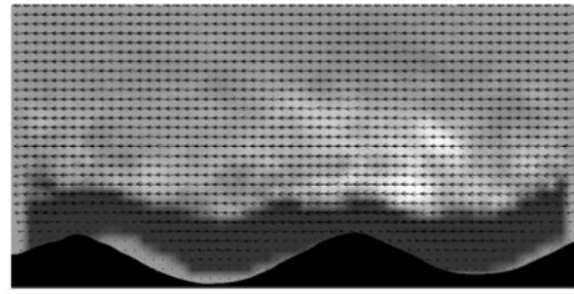

$\phi=0^{\circ}$ (wave crest)

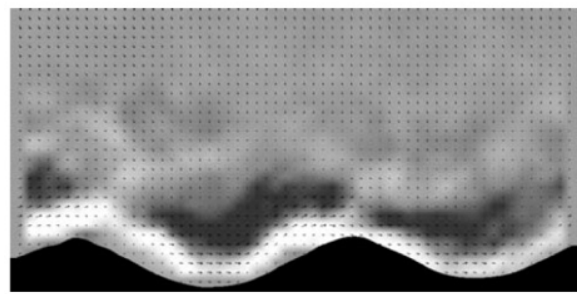

$\phi=90^{\circ}$ (reversing flow)

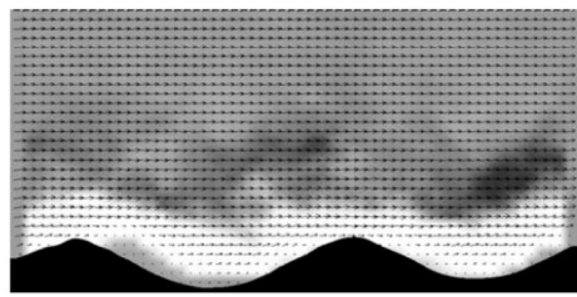

$\phi=180^{\circ}$ (wave trough)

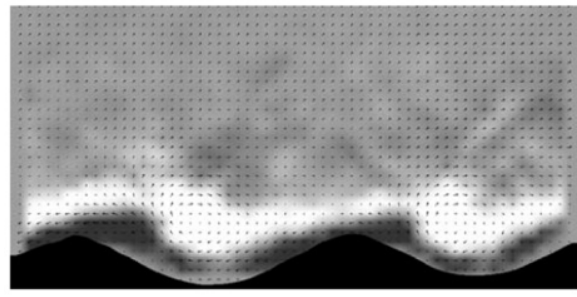

$\phi=270^{\circ}$ (reversing flow)

$\rightarrow$

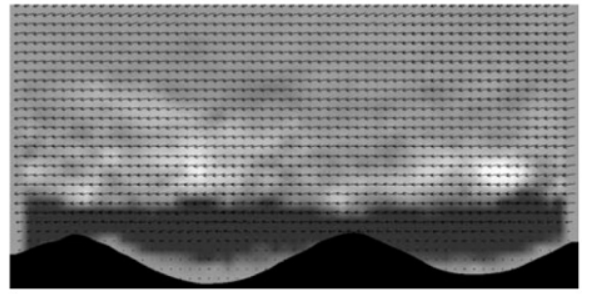

$\phi=45^{\circ}$

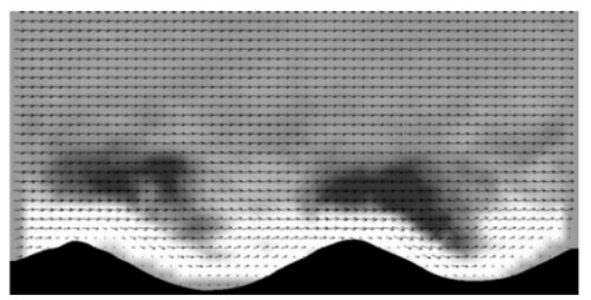

$\phi=135^{\circ}$

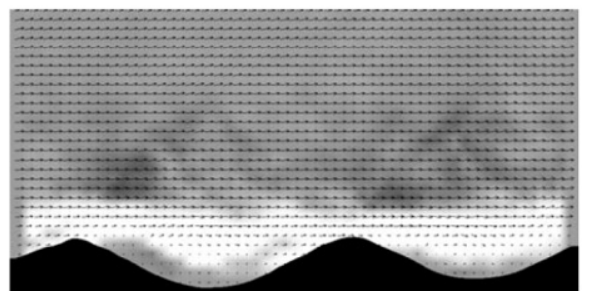

$\phi=225^{\circ}$

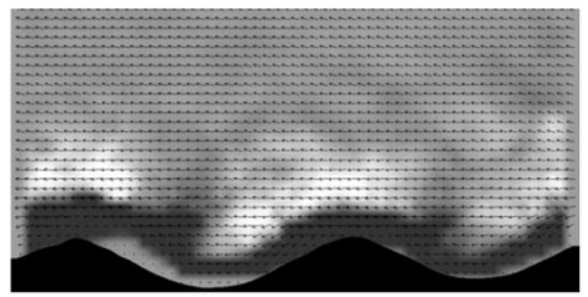

$\phi=315^{\circ}$

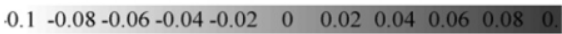

Fig. 11. Equilibrium ripples. Phase-averaged velocity-vector and vorticity maps (waves travelling right to left, ripple length $=0.042 \mathrm{~m}$ ). Respective vector and $\hat{\omega}_{y}$ $\left(\mathrm{s}^{-1} \times 10^{3}\right)$ scales are highlighted.

the strong near-bed vortices are weaker ones of opposite sign, which are remnants of the dominant vortices from the previous half-cycles. An insightful discussion of the trajectories of these vortices above equilibrium ripples (including hysteresis based on varying initial bed states) is given by Admiraal et al. (2006). Away from the bed, the flow is essentially rotationfree, approaching the prediction of plane-bed wave theory. 


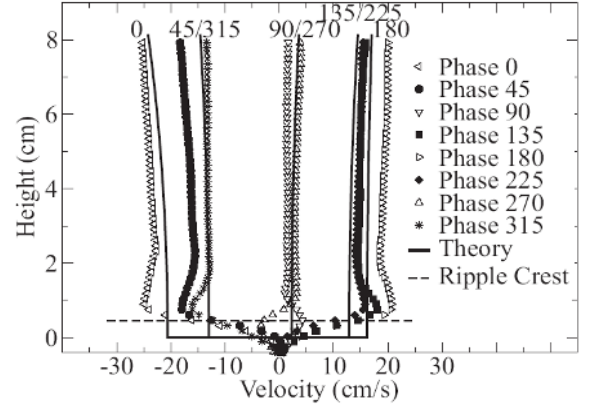

(a)

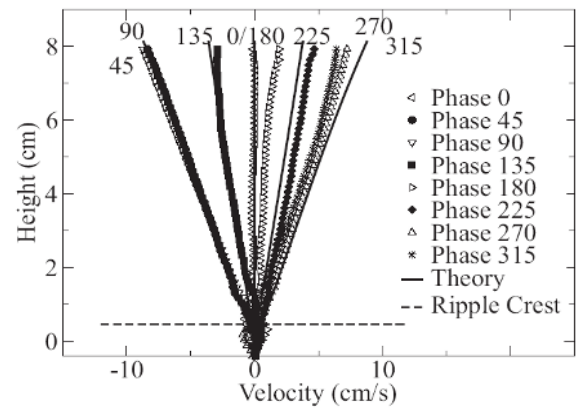

(b)

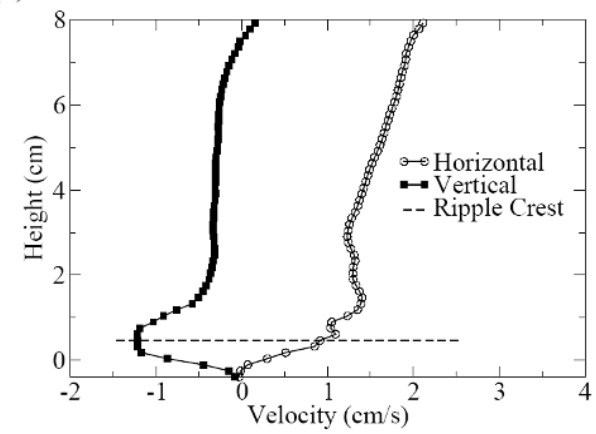

(c)

Fig. 12. Equilibrium ripples. Phase- and spatially-averaged (a) horizontal velocity $\langle\hat{u}\rangle$ and (b) vertical velocity $\langle\hat{w}\rangle$ (labelled by phase, with second-order plane-bed theoretical values shown as "Theory"), and (c) time- and spatially-averaged velocities $\langle\bar{u}\rangle$ and $\langle\bar{w}\rangle$. "Ripple Crest" shown is at equilibrium. "Height" is relative to mean bed level.

\subsection{Velocities}

Phase- and spatially-averaged horizontal and vertical velocity variations with wave phase are shown in Figs. 12a and 12b, respectively. Also shown are the theoretical (plane-bed) values for the velocity, namely (36) and (37), given by the second-order Stokes' theory. It can be seen from Fig. 12 that both $\langle\hat{u}\rangle$ and $\langle\hat{w}\rangle$ are larger near the bed than predicted from plane-bed theory, because of the formation of vortex structures resulting from the presence of the ripples. Away from the bed, the phase-averaged velocities approximate theoretical plane-bed-flow expectations.

The double-averaged (in time and space) horizontal velocities of Fig. 12c comprise a slight residual drift velocity arising from minor recirculation within the wave-flume, together with averaged near-bed asymmetric-vortexinduced velocities that are offshore and onshore above and below the ripple crest respectively (e.g., Davies and Villaret 1999). The general near-bed drift velocity is offshore (towards the wavemaker) and in the opposite direction to 
wave propagation (Fig. 12c), other authors having noted a similar drift velocity pattern (e.g., Liu et al. 2004). As indicated in Section 4.3, the accentuated negative near-bed vertical-velocity estimates of Fig. 12c reflect the artificial effect of the sediment settling velocity.

Figure 13 presents spatially-varying time-averaged velocities, with the velocities of Fig. 12c having been subtracted. The symmetry of Fig. 13 is notable, with two steady-streaming contra-rotating cells per wavelength (similar to those obtained by other authors for vortex ripples, e.g., Huang and Dong 2002, Rousseaux et al. 2004) acting to move sediment from the trough to the crest, and thereby promoting ripple growth and stability. The velocity fields shown in this figure highlight "coherent" organized structures in mean-flow disturbances introduced by the ripples. For the present orbital ripples, the time-averaged flow structure, $\bar{u}_{i}=\left\langle\bar{u}_{i}\right\rangle+\tilde{u}_{i}$, then consists of a pair of counter-rotating vortices set up by the ripples (Fig. 13), superimposed on the overall time- and spatially-averaged flow (Fig. 12c).

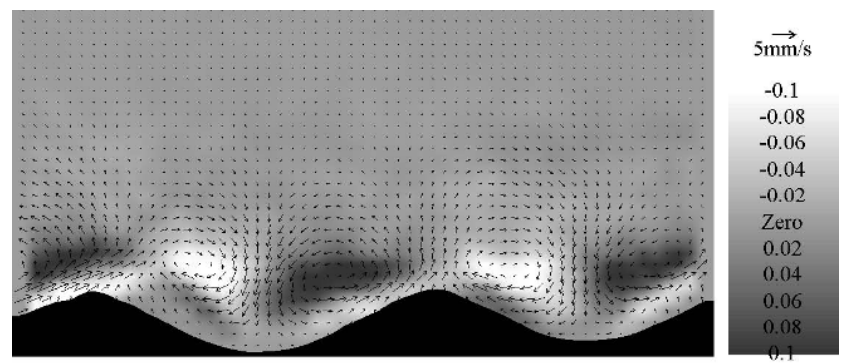

Fig. 13. Equilibrium ripples. Time-averaged vectors (drift velocities removed, ripple length $=0.042 \mathrm{~m})$. Vorticity $\hat{\omega}_{y}\left(\mathrm{~s}^{-1} \times 10^{3}\right)$ contours are highlighted.

\subsection{Stresses}

Spatially-averaged shear stresses are shown in Fig. 14, where (a) and (b) involve time-averaging and phase-averaging, respectively. Comparison of the total time- and spatially-averaged stress for the bed of equilibrium ripples with that for the plane-bed at the start of the experiment (Fig. 14a) highlights that bed-induced effects on momentum flux extend to approximately six ripple amplitudes $\left(6 a_{e}\right)$ above the mean bed level. This near-bed perturbation is superimposed on a small-magnitude variation in total momentum flux over the flow depth (Fig. 14a).

For the time- and spatially-averaged shear stress (Fig. 14a), the individual terms of (32) are shown to illustrate their relative importance. This stress is dominated by the 4 th term, $-\rho \overline{\langle\hat{u}\rangle\langle\hat{w}\rangle}$, consistent with the indications of Nielsen (1992), this wave-phase stress (arising from correlations in phase velocities) reflecting the centrality to the momentum flux of large-scale cir- 


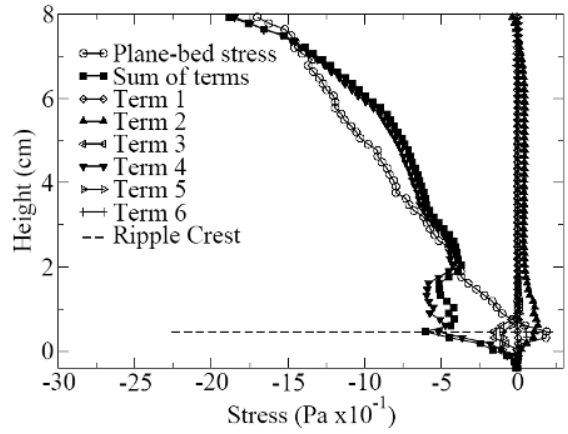

(a)

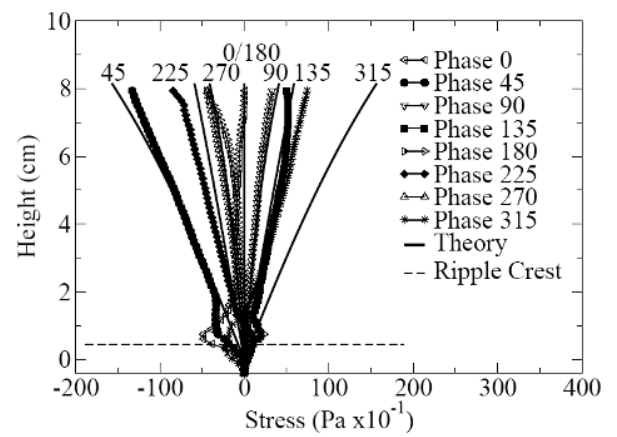

(b)

Fig. 14. Equilibrium ripples. Spatially-averaged shear stress for equilibrium ripples: (a) time averaged $\langle\bar{\tau}\rangle$ (Terms are defined in (32), with measured total stress above the plane bed shown for comparison), and (b) phase-averaged total $\langle\hat{\tau}\rangle$ (with "Theory" from (38) for plane-bed conditions). "Ripple Crest" shown is at equilibrium. "Height" is relative to mean bed level.

culations produced by wave-induced orbital motions. Form-induced stresses (Terms 3 and 5) also become significant in transferring momentum within a ripple height of the ripple crest. Term 2 (representing convective transfer of momentum) becomes notable as the bed is approached, although this variation reflects the artificial effect of the sediment settling velocity. The turbulent component of momentum flux (Term 6), involving fluctuating velocities, is found to be minor in comparison, consistent with previous research for plane-bed oscillatory flows over rough beds (Sleath 1987).

The total time- and spatially-averaged shear stress (Fig. 14a) is of a lesser magnitude than total phase- and spatially-averaged shear stresses occurring over a wave cycle (Fig. 14b). The outer-flow phase- and spatiallyaveraged shear stress can be seen to vary at double the frequency of the waves generating the flows. This reflects the dominance of the wave-phase stress, Term 4 of (31), in total stresses, this stress arising from the correlation of horizontal and vertical phase-averaged velocities that are approximately $90^{\circ}$ out of phase.

For phase- and spatially-averaged stresses occurring over a wave cycle, the individual terms of (31) are shown in Fig. 15 to illustrate their relative importance. As for the time- and spatially-averaged stresses of Fig. 14a, phase- and spatially-averaged shear stresses are dominated by the wavephase stress (Term 4, arising from correlations in phase velocities), particularly for phases of larger stresses $\left(45^{\circ}, 135^{\circ}, 225^{\circ}\right.$, and $\left.315^{\circ}\right)$, confirming the centrality to the momentum flux of large-scale circulations produced by wave-induced orbital motions. Term 2 (representing convective transfer of momentum) is also significant throughout the depth of flow, particularly for 

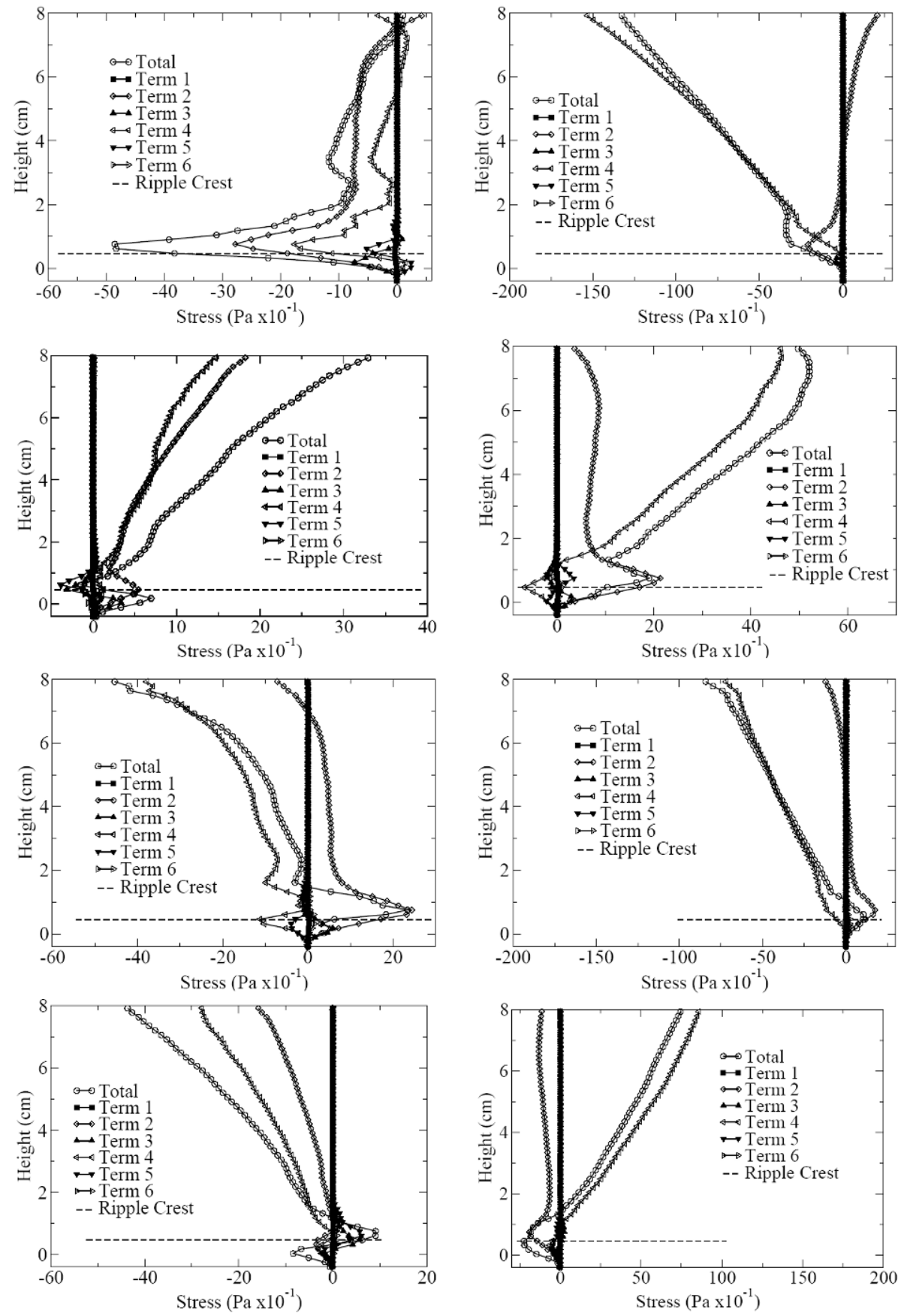

Fig. 15. Equilibrium ripples. Phase- and spatially-averaged shear stress $\langle\hat{\tau}\rangle$. Phases clockwise from top-right: $45^{\circ}, 135^{\circ}, 225^{\circ}, 315^{\circ}, 270^{\circ}, 180^{\circ}, 90^{\circ}$, and $0^{\circ}$, with Terms defined in (31). "Ripple Crest" shown is at equilibrium. "Height" is relative to mean bed level. 
phases of lower stresses $\left(0^{\circ}, 90^{\circ}, 180^{\circ}\right.$, and $\left.270^{\circ}\right)$, and also near the bed, although the near-bed variations reflect the artificial effect of the sediment settling velocity. Form-induced stresses (Terms 3 and 5) are again notable in transferring momentum within a ripple height of the ripple crest, with the turbulent (Term 6) and viscous (Term 1) components of momentum flux effectively negligible throughout the flow depth. Recognising the dominance of the wave-phase terms in the shear-stresses of Fig. 15, theoretical total phase- and spatially-averaged stress was calculated for plane-bed conditions from (38). The results of Fig. 14b generally indicate that away from the bed, the variation of shear stress with phase approximates theoretical plane-bed expectations. Near the bed, differences from plane-bed theory principally reflect the artificial effect of the sediment settling velocity.

\section{CONCLUSIONS}

Spatial-averaging procedures give new forms of fluid continuity and momentum equations for oscillatory flow over rough beds, with the momentum equations naturally and explicitly including the full range of fluid stresses and boundary form and skin friction drag highlighted previously as potentially significant in flow dynamics. The double-averaged (in space and phase or time) equations of mass and momentum conservation are shown to be appropriate for analyses of fixed rough beds or equilibrium ripples. For rapidly-changing bed conditions, e.g., for growing ripples, use of the spatiallyaveraged equations for conservation of mass and momentum is appropriate. Where repeated observations of the changing bed conditions are available, the ensemble and spatially-averaged versions of these equations can be used for more detailed analyses of the flow dynamics. Decomposition and analysis of oscillatory flow within this framework aids data convolution and enables the relative significance of constituent parameters and flow mechanisms to be determined.

From PIV measurements of oscillatory flow over intermediate-depth orbital-vortex ripples growing from plane-bed conditions to equilibrium, plane-bed flow fields are found to parallel wave-theory expectations in terms of velocities, shear stresses and vorticities. As ripples develop, the near-bed flow is shown to cycle between a skimming flow of a strong shear layer following the bed profile for an overhead wave crest or trough, and strong vortex motion between ripple crests as the flow reverses, these respective flows strengthening with ripple growth. Magnitudes of near-bed phase-averaged vorticity and shear stress reflect the strength of the near-bed shear layer relative to that of local vortex structures for flow over orbital-vortex ripples. For larger ripples, additional alternating-sign lesser-magnitude vorticity peaks appear higher in the flow, reflecting the vortices ejected into the flow with the previous flow reversals. In terms of momentum flux components, both 
the time- and spatially-averaged stress and the phase-averaged equivalent are dominated by the contribution from the wave-phase term, reflecting the significance to the momentum flux of large-scale circulations produced by wave-induced orbital motions. For equilibrium ripples, the form-induced components are also notable in the vicinity of the ripple crest. Turbulent and viscous components of momentum flux are found to be effectively negligible throughout the flow depth. The time-averaged flow structure for equilibrium ripples consists of a pair of counter-rotating vortices within each ripple length, superimposed on overall time- and spatially-averaged velocity profiles. These ripple-induced recirculating cells act to move sediment from the trough to the crest, and thereby promote ripple growth and stability.

Acknowledgements. This research was partly funded by the Marsden Fund (UOA220) administered by the Royal Society of New Zealand. The writers gratefully acknowledge the review comments of Eric Barthélemy, Peter Nielsen, and Tom O'Donoghue that have acted to strengthen the paper.

\section{References}

Admiraal, D., R. Musalem-Jara, M. García, and Y. Niño (2006), Vortex trajectory hysteresis above self-formed vortex ripples, J. Hydrol. Res. 44, 4, 437-450.

Blondeaux, P. (2001), Mechanics of coastal forms, Ann. Rev. Fluid Mech. 33, 339370.

Coleman, S.E., and V.I. Nikora (2008), A unifying framework for particle entrainment, Water Resour. Res. 44, W04415, DOI: 10.1029/2007WR006363.

Coleman, S.E., E. Schlicke, and S. Blackbourn (2005), Growth of wave-induced ripples, Proc. 4th IAHR Symposium on River, Coastal and Estuarine Morphodynamics, Urbana, IL, 4-7 October, 963-971.

Davies, A.G., and C. Villaret (1999), Eulerian drift induced by progressive waves above rippled and very rough beds, J. Geophys. Res. 104, C1, 1465-1488.

Davis, J.P., D.J. Walker, M. Townsend, and I.R. Young (2004), Wave-formed sediment ripples: transient analysis of ripple spectral development, J. Geophys. Res. 109, C07020, 15 pp.

Dean, R.G., and R.A. Dalrymple (1991), Water wave mechanics for engineers and scientists, Advanced Series on Ocean Engineering 2, World Scientific Publ. Co., Singapore.

Faraci, C., and E. Foti (2002), Geometry, migration and evolution of small-scale bedforms generated by regular and irregular waves, Coastal Engineering 47, 35-52. 
Giménez-Curto, L.A., and M.A. Corniero Lera (1996), Oscillating turbulent flow over very rough surfaces, J. Geophys. Res. 101, C9, 20,745-20,758.

Hsu, T.-J., T. Sakakiyama, and P.L.-F. Liu (2002), A numerical model for wave motions and turbulent flows in front of a composite breakwater, Coastal Engineering 46, 25-50.

Huang, C.-J., and C.-M. Dong (2002), Propagation of water waves over rigid rippled beds, J. Waterway, Port, Coastal, and Ocean Engrg. 128, 5, 190-201.

Hughes, S.A. (1993), Physical models and laboratory techniques in coastal engineering, Advanced Series on Ocean Engineering 7, World Scientific Publ. Co., Singapore.

Isaacson, M. (1991), Measurement of regular wave reflection, J. Waterway, Port, Coastal, and Ocean Engrg. 117, 6, 553-569.

Liu, P.L.-F., K.A. Al-Banaa, and E.A. Cowen (2004), Water wave induced boundary layer flows above a rippled bed. In: 'PIV and Water Waves', Advances in Coastal and Ocean Engineering 9, World Scientific Publ. Co., Singapore.

Lofquist, K. (1978), Sand Ripple Growth in an Oscillatory-Flow Water Tunnel, Technical Paper 78-5, Coastal Engineering Research Center, US Army Corps of Engineering, $101 \mathrm{pp}$.

Longuet-Higgins, M.S. (1997), Progress toward understanding how waves break, Proc. 21st Symposium on Naval Hydrodynamics, Trondheim, Norway, June 1996, National Academy Press, Washington, DC.

Lowe, R.J., J.R. Koseff, and S.G. Monismith (2005a), Oscillatory flow through submerged canopies: 1. Velocity structure, J. Geophys. Res. 110, C10016, $17 \mathrm{pp}$.

Lowe, R.J., J.R. Koseff, S.G. Monismith, and J.L. Falter (2005b), Oscillatory flow through submerged canopies: 2. Canopy mass transfer, J. Geophys. Res. 110, C10017, 14 pp.

Nielsen, P. (1992), Coastal Bottom Boundary Layers and Sediment Transport, Advanced Series on Ocean Engineering 4, World Scientific Publ. Co., Singapore.

Nikora, V.I., D.G. Goring, I. McEwan, and G. Griffiths (2001), Spatially-averaged open-channel flow over a rough bed, J. Hydraul. Engrg. ASCE 127, 2, 123-133.

Nikora, V.I., I. McEwan, S.R. McLean, S.E. Coleman, D. Pokrajac, and R. Walters (2007), Double-averaging concept for rough-bed open-channel and overland flows: Theoretical background, J. Hydraul. Engrg. ASCE 133, 8, 873883 .

Ranasoma, K.I.M., and J.F.A. Sleath (1992), Velocity measurements close to rippled beds, Proc. 23rd Int. Conf. Coastal Engrg., American Society of Civil Engineers, 2383-2396. 
Raudkivi, A.J. (1998), Loose Boundary Hydraulics, 4th ed., A.A. Balkema, The Netherlands, $512 \mathrm{pp}$.

Rousseaux, G., H. Yoshikawa, A. Stegner, and J.E. Wesfreid (2004), Dynamics of transient eddy above rolling-grain ripples, Phys. Fluids 16, 4, 1049-1058.

Schlicke, E., S.E. Coleman, and V.I. Nikora (2005), A PIV investigation into the interaction between wave motion and sediment ripples, 4th IAHR Symposium on River, Coastal and Estuarine Morphodynamics, Urbana, IL, 981-989.

Schlicke, E., S.M. Cameron, and S.E. Coleman (2007), Galvanometer-based PIV for liquid flows, Flow Measurement and Instrumentation 18, 27-36.

Sleath, J.F.A. (1987), Turbulent oscillatory flow over rough beds, J. Fluid Mechanics 182, 369-409.

Svendsen, I.A., and I.G. Jonsson (1976), Hydrodynamics of Coastal Regions, Technical University of Denmark.

van der Werf, J.J., J.S. Doucette, T. O’Donoghue, and J.S. Ribberink (2007), Detailed measurements of velocities and suspended sand concentrations over full-scale ripples in regular oscillatory flow, J. Geophys. Res. 112, F02012, DOI: $10.1029 / 2006 J F 000614$.

Voropayev, S.I., G.B. McEachern, D.L. Boyer, and H.J.S. Fernando (1999), Dynamics of sand ripples and burial/scouring of cobbles in oscillatory flow, $\mathrm{Ap}$ plied Ocean Res. 21, 5, 249-261.

Whitaker, S. (1999), The Method of Volume Averaging, Kluwer Academic Publishers, Dordrecht.

Received 27 July 2007

Accepted 27 March 2008 